\title{
KINEMATICALLY OPTIMAL ROBUST CONTROL OF REDUNDANT MANIPULATORS
}

\author{
M. GALICKI \\ Faculty of the Mechanical Engineering \\ University of Zielona Góra \\ ul. Prof. Z. Szafrana 4, 65-516, Zielona Góra, POLAND \\ E-mail: M.Galicki@ibem.uz.zgora.pl
}

\begin{abstract}
This work deals with the problem of the robust optimal task space trajectory tracking subject to finite-time convergence. Kinematic and dynamic equations of a redundant manipulator are assumed to be uncertain. Moreover, globally unbounded disturbances are allowed to act on the manipulator when tracking the trajectory by the endeffector. Furthermore, the movement is to be accomplished in such a way as to minimize both the manipulator torques and their oscillations thus eliminating the potential robot vibrations. Based on suitably defined task space non-singular terminal sliding vector variable and the Lyapunov stability theory, we derive a class of chattering-free robust kinematically optimal controllers, based on the estimation of transpose Jacobian, which seem to be effective in counteracting both uncertain kinematics and dynamics, unbounded disturbances and (possible) kinematic and/or algorithmic singularities met on the robot trajectory. The numerical simulations carried out for a redundant manipulator of a SCARA type consisting of the three revolute kinematic pairs and operating in a two-dimensional task space, illustrate performance of the proposed controllers as well as comparisons with other well known control schemes.
\end{abstract}

Key words: redundant robotic manipulator, task space trajectory tracking, robust optimal finite-time control, Lyapunov stability.

\section{Introduction}

Redundant robotic manipulators have been widely employed for decades in industry. They have found a broad area of applications which include e.g. inserting a shaft into a bearing hole or an assembly of electronic components onto the small surface of printed circuit boards. These tasks require, by their nature, extremely high precision and stability of the performance. In order to fulfil the aforementioned requirements, control algorithms should take into account the following factors: uncertainties of both kinematic and dynamic equations and external disturbances. However, robot dynamic parameters, the lengths of the links, joint offsets and the object or tool which the end-effector is holding, are never known exactly in practice. Therefore, it is particularly important to design control strategies that ensure accurate, optimal and fast convergence to the stable equilibrium despite the existence of the aforementioned factors. In most situations met in practice, those tasks are specified in terms of a trajectory expressed in Cartesian (task) coordinates to be tracked by the end-effector. On the other hand, redundant degrees of freedom make it possible to optimally achieve some useful objectives such as collision avoidance in the work space with obstacles, joint limit avoidance and/or reaching some rest (preferred) posture. Thus, a controller to be designed for kinematically redundant manipulators should both accurately track desired end-effector trajectory (primary task) and optimally accomplish additive objectives (secondary task) subject to uncertain both kinematic and dynamic equations and external disturbances. Due to the challenging nature of the aforementioned control design problems, many researchers have proposed different types of controllers. In such a context, one can distinguish a few approaches of controlling the kinematically redundant manipulators. 
The first approach presented in works Khatib [1], Hsu et al. [2], Canudas et al. [3], Siciliano et al. [4], Galicki [5], Kelly and Moreno [6], Nakanishi et al. [7], Moreno-Valenzuela and Gonzales-Hernandez [8], Tatlicioglu et al. [9], Sadeghian et al. [10], Sadeghian et al. [11], Feng and Palaniswami [12], Zergeroglu et al. [13], Braganza et al. [14], Braganza et al. [15], Galicki [16], Cheah et al. [17], Li and Cheah [18], Li and Cheah [19], Galicki [20] is based on the application of the (generalized) pseudo-inverse of the manipulator Jacobian matrix in the control formulation. The control techniques offered in Khatib [1], Hsu et al. [2], Canudas et al. [3], Siciliano et al. [4], Galicki [5], Kelly and Moreno [6], Nakanishi et al. [7], Moreno-Valenzuela and Gonzales-Hernandez [8] require the full knowledge of the robot dynamics neglecting the external disturbances. Works Tatlicioglu et al. [9], Sadeghian et al. [10], Sadeghian et al. [11], Feng and Palaniswami [12], Zergeroglu et al. [13], Braganza et al. [14], Braganza et al. [15], Galicki [16], Cheah et al. [17], Li and Cheah [18], Li and Cheah [19], Galicki [20] present adaptive control algorithms to compensate for parametric uncertainties in dynamic model including only the linearly parametrizable friction terms (viscous friction) and also neglecting the external (non-linearly parametrizable) disturbances. Moreover, control laws from Tatlicioglu et al. [9], Sadeghian et al. [10], Sadeghian et al. [11], Feng and Palaniswami [12], Zergeroglu et al. [13], Braganza et al. [14], Braganza et al. [15], Galicki [16], Cheah et al. [17], Li and Cheah [18], Li and Cheah [19] use pseudo-inverse of either the exact or approximate Jacobian matrices. Our recent study Galicki [20] estimates the pseudo-inverse by some non-singular matrix which is adaptively computed. Model based robust control schemes were investigated in works Zergeroglu et al. [21], Ozbay et al. [22], Singh and Sukavanam [23]. The controllers proposed in Zergeroglu et al. [21], Ozbay et al. [22] ensure uniformly ultimately bounded end-effector and sub-task tracking despite the parametric uncertainties associated with the dynamic equations, an upper bound on the parameter accuracy and globally bounded external disturbances. A neural network based adaptive asymptotically stable control scheme, in the presence of model uncertainties and globally bounded external disturbances, has been designed in Singh and Sukavanam [23]. Although control algorithms based on the pseudo-inverse of the Jacobian matrix are attractive and further investigated by many researchers, they posses some shortcomings. Namely, generated controls provide only sub-optimal (and not optimal) solutions. Moreover, pseudo-inverse control strategies are not, in general, repeatable Shamir and Yomdin [24], Roberts and Maciejewski [25]. Consequently, an important class of cyclic technological operations (cyclic kinematic tasks) can not be accomplished by this approach. Furthermore, almost all those trajectory tracking algorithms require explicitly inverse or pseudo-inverse of a Jacobian matrix or its estimate which can potentially contain kinematic and/or algorithmic singularities. As a consequence, these algorithms can cause the control inputs to become unbounded what leads to vibrations of the robot. In order to tackle the singular configurations, the use of damped least-squares has been proposed in works Nakamura and Hanafusa [26], Wampler and Leifer [27] in lieu of the pseudo-inverses. Nevertheless, this technique suffers from the tracking errors due to a long-term numerical integration drift. Moreover, all the control schemes assume globally bounded disturbances when tracking the trajectory whereas e.g. a viscous friction term is globally unbounded with respect to joint velocity. The assumption of global boundedness of external disturbances may lead to deterioration of the accuracy of trajectory tracking. Finally, aforementioned controllers provide only at most asymptotic stability what may be insufficient for accomplishment of tasks requiring the extremely high precision (e.g. assembly of electronic components on the small surface of printed circuit boards). The second approach presented in only few works Seraji and Colbaugh [28], Peng and Adachi [29], Ott et al. [30], Oh and Chung [31], Colbaugh and Glass [32] is based on utilizing augmented task space techniques such as the extended Jacobian Balleieul [33] or the configuration control proposed by Seraji and Colbaugh [28]. In this approach, the dimension of the operational space is extended by incorporating as many additional constraints as the degrees of redundancy, and hence, the resulting system becomes non-redundant. The control algorithms from Seraji and Colbaugh [28], Peng and Adachi [29], Ott et al. [30] require exact dynamic model thus guaranteeing the asymptotic tracking. Using extended Jacobian, nominal values of the parameters of dynamic equations and the momentum feed-back disturbance observer, a trajectory tracking control law has been proposed in Oh and Chung [31] without its stability analysis. Work Colbaugh and Glass [32] presents an adaptive scheme for the motion control of kinematically redundant manipulator subject to parametric uncertainties of dynamic equations and globally bounded disturbances. In the absence of disturbances and under some sufficient conditions on gain parameters, control law from Colbaugh and Glass [32], is shown to 
ensure only globally ultimately bounded Cartesian tracking error. The control formulations based on the augmented task space technique posses some drawbacks. The controllers offered in Seraji and Colbaugh [28], Peng and Adachi [29], Ott et al. [30], Oh and Chung [31] require inverse of the extended Jacobian matrix which can potentially contain algorithmic and/or kinematic singularities Balleieul [33] and as a consequence, can cause the control inputs to become unbounded even though the manipulator is not in a singular configuration. Moreover, due to adaptive estimations of the dynamic equations terms, control law presented in Colbaugh and Glass [32] seems to be very time consuming and is only stable in the presence of bounded disturbances which may significantly worsen the tracking accuracy. The third approach, presented in several papers Wolovich and Elliot [34], Siciliano [35], Kelly [36], Cheah [37], Cheah et al. [38], Moosavian and Papadopoulos [39] that include its stability analysis, is based on the use of the transpose of the Jacobian. Nevertheless, work Wolovich and Elliot [34] guarantees only ultimate boundedness provided that time-derivative of the desired trajectory is globally norm bounded. The asymptotically stable purely kinematic control scheme offered in Siciliano [35] indeed eliminates explicit computation of the inverse but introduces undesirable chattering effect. In work Cheah [37], it is claimed that controllers based on transposed Jacobian and inverse Jacobian are dual in the sense that the transformation from task space to joint space can be either defined as transposed Jacobian or inverse Jacobian. As was also shown in Cheah [37], Cheah et al. [38], approximate transpose Jacobian control law is asymptotically stable. In work Moosavian and Papadopoulos [39], a modified transpose Jacobian algorithm was developed which employs stored data of the control command in the previous time step, as a learning tool to yield an improved performance. Nevertheless, works Kelly [36], Cheah [37], Cheah et al. [38], Moosavian and Papadopoulos [39] have shown stability of the performance for the set-point control problems.

In this paper, a new task space non-singular terminal sliding manifold (TSM) of the first order is introduced to optimally track the end-effector trajectory of redundant manipulators. As is known Edwards and Spurgeon [40], Utkin [41], sliding mode is accurate and insensitive to disturbances. However, the main drawback of the standard first order sliding modes is mostly related to the undesirable chattering effect Fridman [42]. In order to eliminate (or to alleviate) the chattering effect, the robust sliding mode controllers, expressed both in a joint and task coordinates space and based on the second-order sliding techniques, have been used in robotic applications Galicki [43], Galicki [44], Galicki [45]. Nevertheless, the second- and higher-order sliding techniques can not be applied to discontinuous disturbances (e.g. to the Coulomb or Stribeck terms Haessing and Friedland [46], Wit et al. [47]) and/or to continuous and everywhere non-differentiable disturbances (e.g. to a Brownian motion) acting on the robotic manipulator. Application of filtered techniques to smooth out the control signals introduces additional phase delay related with recursive lowpass filters which may further limit the performance of a closed-loop system. An approach based on application of the Newton predictor enhanced Kalman filter (NPEKF) which provides a wide bandwidth and somewhat reduces phase lag, has been proposed in Han [48]. Motivated in part by the computed torque approach Spong and Vidyasagar [49], Siciliano et al. [4], we introduce a new class of robust optimal controllers based on an estimate of the transpose Jacobian. By fulfilment of a reasonable assumption regarding the estimate of the extended Jacobin matrix, the proposed control scheme is shown to be finite-time stable despite uncertain both kinematic and dynamic equations and globally unbounded disturbances acting on the manipulator. The advantages of the estimated Jacobian transpose approach are that it is computationally efficient and it does not suffer from singularity problem. Moreover, the proposed control laws ensure both optimal solution and a cyclic joint trajectory provided the desired end-effector trajectory is cyclic. As opposed to other existing robust control algorithms, our approach both reduces the values of torques by minimizing a suitably chosen objective function and eliminates their oscillations by introducing a boundary layer technique. The boundary layer technique, introduced herein to eliminate undesirable chattering effect, guarantees (without increase of the controller gains) the uniform convergence of the task errors to the origin as boundary layer size tends to approach zero. The remainder of the paper is organised as follows. Section 2 formulates the finite-time trajectory tracking task. Section 3 sets up a class of task space robust optimal controllers solving the trajectory tracking problem in a finite-time subject to uncertain both robot kinematic and dynamic equations as well as unbounded disturbances. Section 4 presents computer examples of the end-effector trajectory tracking by a redundant manipulator of a SCARA type, consisting of three revolute kinematic pairs and operating 
in a two-dimensional task space. Finally, some concluding remarks are drawn in Section 5. Throughout this paper, $\lambda_{\max }(\cdot)$ denotes the maximal eigenvalue of the matrix $(\cdot)$.

\section{Problem formulation}

The robust control scheme designed in the next section is applicable to holonomic fully actuated mechanical systems comprising redundant manipulators considered here which are described, in general, by the following dynamic equations, expressed in generalized (joint) coordinates $q=\left(q_{1}, \ldots, q_{n}\right)^{T}$ Spong and Vidyasagar [49]

$$
M(q) \ddot{q}+C(q, \dot{q}) \dot{q}+G(q)+D(t, q, \dot{q})=v,
$$

where $n$ is the number of kinematic pairs of the V-th class; $\dot{q}$ and $\ddot{q}$ represent velocity and acceleration, respectively expressed in generalized coordinates; $M(q)$ denotes the $n \times n$ positive definite, symmetric inertia matrix; $C(q, \dot{q}) \dot{q}$ is the $n$-dimensional vector representing centrifugal and Coriolis forces; $v=\left(v_{1}, \ldots, v_{n}\right)^{T}$ stands for the $n$-dimensional vector of controls (torques/forces); $G(q)$ is the $n$-dimensional vector of generalized gravity forces; $D(t, q, \dot{q})$ means the $n$-dimensional external disturbance signal which is (by assumption) at least a locally bounded Lebesgue measurable mapping. Without loss of generality, $D$ is assumed to be upper estimated as follows

$$
\|D\| \leq \alpha_{0}(t, q, \dot{q})
$$

where \|\| denotes Euclidean norm; $\alpha_{0}(\cdot)$ stands for the time dependent known non-negative locally bounded Lebesgue measurable function. In what follows, $D$ is assumed in further analysis to represent both the friction in the sliding (viscous) and in the presliding (also called stiction) regime Haessing and Friedland [46], Wit et al. [47]. In such a case, $D$ equals

$$
D=d_{0}(q-q(0))+d_{1} \dot{q}+d_{2} \operatorname{sign}(\dot{q})+d_{3} \exp \left(-d_{4}\|\dot{q}\|^{2}\right) \operatorname{sign}(\dot{q})
$$

where $q(0)$ means initial posture of the manipulator; $d_{0}$ denotes a stiffness coefficient for the position-friction torque relationship at joint velocity reversal; $d_{1}$ is a viscous damping coefficient; $d_{2}$ stands for the Coulomb coefficient and $d_{3}, d_{4}$ are constants representing the Stribeck effect. Consequently,

$$
\alpha_{0}=\overline{d_{0}}\|q-q(0)\|+\overline{d_{1}}\|\dot{q}\|+\overline{d_{2}}+\overline{d_{3}} \exp \left(-\underline{d_{4}}\|\dot{q}\|^{2}\right)
$$

where $\overline{d_{0}}, \ldots, \overline{d_{3}}$ stand for upper estimations of unknown coefficients $d_{0}, \ldots, d_{3}$ and $d_{4}$ denotes lower estimate of the Stribeck parameter $d_{4}$, respectively.

The direct kinematic mapping between joint coordinates $q$ of the manipulator and the end-effector coordinates $p_{e}=\left(p_{e, 1}, \ldots, p_{e, m}\right)^{T} \in \mathbb{R}^{m}$ can be written as

$$
p_{e}=f_{e}(q, X)
$$


where $m$ is the dimension of the Cartesian space in which the end-effector operates; $f_{e}: \mathbb{R}^{n} \times \mathbb{R}^{k} \rightarrow \mathbb{R}^{m}$ denotes the $m$-dimensional non-linear (with respect to $q$ ) mapping constructed from the kinematic equations of the manipulator; $f_{e}=\left(f_{e, 1}, \ldots, f_{e, m}\right)^{T} ; X=\left(X_{1}, \ldots, X_{k}\right)^{T}$ stands for an ordered set of kinematic parameters such as link lengths and/or joint offsets; $k$ denotes the number of kinematic parameters. Moreover, there exist upper $X_{u}$ and lower $X_{l}$ bounds, respectively such that

$$
X_{l} \leq X \leq X_{u}
$$

On account of the redundant manipulator considered herein, the relation $n>m$ holds. Its consequence is a possibility to augment the end-effector conventional trajectory tracking (primary task) with additional user-specified useful task coordinates $p_{a}=\left(p_{a, 1}, \ldots, p_{a, n-m}\right)^{T} \in \mathbb{R}^{n-m}$ (secondary task) of the following general form

$$
p_{a}=f_{a}(q, X),
$$

where

$f_{a}: \mathbb{R}^{n} \times \mathbb{R}^{k} \rightarrow \mathbb{R}^{n-m}$ is a given at least twice differentiable mapping with respect to $q ; f_{a}=\left(f_{a, 1}, \ldots, f_{a, n-m}\right)^{T}$. From the practical point of view, it is particularly important to generate joint trajectory $q=q(t)$ in such a way as to minimize an objective function $\mathcal{A}(q, X)$ subject to constraints Eq.(2.5). The objective function $\mathcal{A}$ may be any at least twice differentiable mapping representing a measure of some kinematic characteristic of the manipulator performance so that the redundant degrees of freedom are exploited to fulfil additional goals: a singularity avoidance, posture control, obstacle avoidance, etc. Yoshikawa [50], Maciejewski and Klein [51]. The general form for $f_{a}$, proposed, e.g., in works Balleieul [33], Perdereau et al. [52] may be expressed as

$$
f_{a}=\mathcal{N}(q, X) \frac{\partial \mathcal{A}(q, X)}{\partial q}
$$

where $\mathcal{N}$ stands for the $(n-m) \times n$ orthogonal complementary matrix to $\jmath(q, X)=\frac{\partial f_{e}(q, X)}{\partial q}$, i.e., $\jmath \mathcal{N}^{T}=0$. Without loss of generality, we shall employ a simple and practically useful optimization criterion for redundancy resolution with a cost function

$$
\mathcal{A}(q)=\frac{c_{\mathcal{A}}}{2}\left\langle q-q_{\text {rest }}, K_{\mathcal{A}}\left(q-q_{\text {rest }}\right)\right\rangle
$$

where $\langle$,$\rangle denotes the scalar product of vectors; c_{\mathcal{A}}$ is a positive constant; $q_{\text {rest }}$ stands for some rest (preferred) posture; $K_{\mathcal{A}}$ is a positive definite diagonal weighting matrix. This criterion has been shown to be useful when creating human-like movement in anthropomorphic robots Cruse et al. [53]. Moreover, for $q_{\text {rest }}=q(0)$, minimization of criterion Eq.(2.9) results in avoiding sudden changes of manipulator configurations and consequently allows to reduce both the values of torques in $L_{2}$ norm and potential robot vibrations by appropriate weighting of joints closer to the robot base. Concatenating Eq.(2.5) with Eq.(2.8) yields the general kinematic and differential mappings between $q$ and extended task coordinates $p=\left(p_{e}^{T} p_{a}^{T}\right)^{T}$ 


$$
p=f(q, X), \quad \dot{p}=J(q, X) \dot{q},
$$

where $f=\left(f_{e}^{T}\left(\mathcal{N} \frac{\partial \mathcal{A}}{\partial q}\right)^{T}\right)^{T}$ and $J=\frac{\partial f}{\partial q}$ is the $n \times n$ extended Jacobian matrix.

A task accomplished by the redundant manipulator consists in tracking both a desired end-effector trajectory $p_{d}^{e}(t) \in \mathbb{R}^{m}, t \in[0, \infty)$ which is assumed to be at least twice continuously differentiable, i.e., $p_{d}^{e}(\cdot) \in C^{2}[0, \infty)$ and desired user specified trajectory $p_{d}^{a}(t) \in \mathbb{R}^{n-m}$ which equals $p_{d}^{a}(t)=0$. By introducing the task tracking error $e=\left(e_{1}, \ldots, e_{n}\right)^{T}=\left(\left(e^{e}\right)^{T}\left(e^{a}\right)^{T}\right)^{T}=f(q, X)-p_{d}(t)$, where $p_{d}=\left(\left(p_{d}^{e}\right)^{T} 0^{T}\right)^{T} ; e^{e}=f_{e}-p_{d}^{e} ; e^{a}=\mathcal{N} \frac{\partial \mathcal{A}}{\partial q}$, we may formally express the task space finite-time control problem by means of the following equations

$$
\begin{aligned}
& \lim _{t \rightarrow T} e(t)=0, \\
& \lim _{t \rightarrow T} \dot{e}(t)=0,
\end{aligned}
$$

where $0 \leq T$ denotes a finite-time of convergence of $f(q)$ to $p_{d} ; e(t)=\dot{e}(t)=0$ for $t \geq T$. Observe that upper equation of Eq.(2.11) presents for $t \geq T$ a necessary condition for minimum of $\mathcal{A}$ subject to equality constraint $f_{e}-p_{d}^{e}=0$. Let us also note that fulfilment of Eq.(2.11) makes a repetitive motion conservative, i.e., closed end-effector trajectory $p_{d}^{e}$ in task space forces the corresponding closed trajectory in the joint space Shamir and Yomdin [24], Roberts and Maciejewski [25]. This property seems to be very useful in accomplishing the cyclic technological operations. Let us rewrite Eq.(2.1) in a more compact form as follows

$$
M(q) \ddot{q}+F(t, q, \dot{q})=v,
$$

where $F=C \dot{q}+G+D$. In the sequel, useful properties of Eq.(2.1), Eq.(2.12) are summarised which will be utilised while designing the controller. The following inequalities are satisfied Spong and Vidyasagar [49]

$$
\begin{aligned}
& 0<\left\|M^{-1}\right\|_{F} \leq \Lambda_{\max }, \\
& \|C \dot{q}\| \leq c_{1}\|\dot{q}\|^{2}, \\
& \|G\| \leq c_{2},
\end{aligned}
$$

where \|\|$_{F}$ means Frobenius (Euclidean) matrix norm; $\Lambda_{\max }, c_{1}, c_{2}$ are known positive scalar coefficients. Based on the properties of Eq.(2.13), one obtains the following upper estimation of $F$

$$
\|F\| \leq \mathcal{E}(t, q, \dot{q}),
$$

where $\mathcal{E}=c_{1}\|\dot{q}\|^{2}+c_{2}+\alpha_{0}$. Moreover, on account of Eq.(2.6), Eq.(2.8), Eq.(2.9) and definition of $J$ in Eq.(2.10), the following inequalities hold true for revolute kinematic pairs 


$$
\begin{aligned}
& \|J\|_{F} \leq c_{3}+c_{4}\left\|q-q_{\text {rest }}\right\|, \\
& \left\|\frac{\partial J}{\partial q}\right\|_{F} \leq c_{5}+c_{6}\left\|q-q_{\text {rest }}\right\|,
\end{aligned}
$$

where $\|J\|_{F}=\max _{X_{l} \leq X \leq X_{u}}\left\{\|J(q, X)\|_{F}\right\} ; c_{3}, \ldots, c_{6}$ are known scalar coefficients. Based on relations Eqs (2.13)-(2.15), the next section will present an approach to the solution of the control problem Eq.(2.1), Eq.(2.11) and Eq.(2.12) making use of the Lyapunov stability theory.

\section{Task space control of the redundant robotic manipulator}

Motivated in part by the static computed torque methodology Spong and Vidyasagar [49], Siciliano et al. [4], we propose now a new control law with transpose estimated Jacobian of the form

$$
v=\hat{J}^{T} u
$$

where $\hat{J}=J(q, \hat{X})$ estimates the uncertain Jacobian matrix $J=J(q, X) ; \hat{X}$ denotes estimation of the unknown kinematic parameters $X ; X_{l} \leq \hat{X} \leq X_{u} ; u \in \mathbb{R}^{n}$ is a new control to be determined. In further analysis, $\hat{J}$ is assumed to be of the full rank in the operation region of the end-effector, i.e.,

$$
\operatorname{rank}(\hat{J})=n
$$

Consequently, from Eq.(3.2), one also obtains that

$$
0<a \mathbb{I}_{n} \leq \hat{J} M^{-1} \hat{J}^{T}
$$

where $a$ denotes estimation of the minimal eigenvalue of matrix $\hat{J} M^{-1} \hat{J}^{T} ; \mathbb{I}_{n}$ stands for the $n \times n$ identity matrix. Let us note that condition Eq.(3.2) may be made somewhat more weaken. It suffices that for $0 \neq x \in \mathbb{R}^{n}$ and singular configuration $q^{\prime}$, the following condition holds true: $x \notin \operatorname{ker}\left(J^{T}\left(q^{\prime}, \hat{X}\right)\right)$. In the sequel, matrix $\hat{J}$ is assumed to fulfil the following inequality

$$
0<\|\hat{J}-J\|_{F} \leq \frac{a \cdot \delta}{\Lambda_{\max } \cdot\|J\|_{F}}
$$

where $\delta$ is a gain coefficient; $\delta \in(0,1)$. Due to minimization of Eq.(2.9) and construction constraints on $q$, which exist for typical industrial manipulators, the maximal value of the right hand side of Eq.(2.15) is finite.

Hence, the minimal value of the right hand side of inequality Eq.(3.4) is strictly positive. In addition, let us note that inequality Eq.(3.4) can be in practice easily fulfilled by selection of a sufficiently accurate device 
for measurement of kinematic parameters $X$ (link lengths, joint offsets). Applying Eq.(3.1) as a control law with transpose estimated Jacobian matrix $\hat{J}$ gives

$$
M \ddot{q}+F=\hat{J}^{T} u=v
$$

Since $M$ is invertible, we obtain

$$
\ddot{q}=M^{-1} \hat{J}^{T} u-M^{-1} F
$$

The objective is to find input signal $u(t)$ and consequently control vector $v(t)$, such that end-effector location vector $p$ follows $p_{d}$. For this purpose, let us twice differentiate $e$ with respect to time thus obtaining

$$
\ddot{e}=J \ddot{q}+\dot{J} \dot{q}-\ddot{p}_{d}
$$

Inserting the right-hand side of Eq.(3.6) into Eq.(3.7), one obtains

$$
\ddot{e}=J M^{-1} \hat{J}^{T} u+Q-\ddot{p}_{d},
$$

where $Q=-J M^{-1} F+\dot{J} \dot{q}$. Furthermore, based on Eq.(2.14) and definition of $Q$ in Eq.(3.8), an upper estimation on $\|Q\|$ takes the form

$$
\|Q\| \leq \mathcal{W}(t, q, \dot{q})
$$

where $\mathcal{W}=\|J\|_{F} \cdot \Lambda_{\max } \cdot \mathcal{E}+\left\|\frac{\partial J}{\partial q}\right\|_{F} \cdot\|\dot{q}\|^{2}$. Let $s=\left(s_{1}, \ldots, s_{n}\right)^{T} \in \mathbb{R}^{n}$ be a task space sliding vector

variable. In order to overcome the limitations and shortcomings of the first order classic sliding variables expressed in joint coordinates Feng et al. [54], Yu et al. [55], Zhao et al. [56], we propose the following nonsingular terminal sliding manifold expressed in the task coordinates

$$
s=\dot{e}+\int_{0}^{t}\left(\lambda_{0} e^{\alpha_{1}}+\lambda_{I}(\dot{e})^{\alpha_{2}}\right) d \tau,
$$

where $\alpha_{1}=\frac{a}{b} ; a, b$ are positive odd numbers, $a<b<2 a ; \quad \alpha_{2}=\frac{2 \alpha_{1}}{1+\alpha_{1}} ; \lambda_{0}=\operatorname{diag}\left(\lambda_{0,1}, \ldots, \lambda_{0, n}\right)$; $\lambda_{1}=\operatorname{diag}\left(\lambda_{1,1}, \ldots, \lambda_{1, n}\right) ; \lambda_{i, j}$ stand for positive coefficients (controller gains); $i=0: 1 ; j=1: n$. In what follows, we give a useful result. 
Lemma 1. If $s=0$ then task errors $(e, \dot{e})$ converge in a finite-time to the origin $(e, \dot{e})=(0,0)$.

Proof. Identity $s=0$ implies equality $\dot{s}=0$. From Eq.(3.10), it follows that

$$
\ddot{e}+\lambda_{0} e^{\alpha_{1}}+\lambda_{l}(\dot{e})^{\alpha_{2}}=0
$$

Expression Eq.(3.11) presents a known homogeneous double integrator system of negative degree equal to $\alpha_{2}-1$. Finite-time stability of homogeneous system Eq.(3.11) was studied e.g. in Bhat and Bernstein [57], Hong [58]. Moreover, the settling-time estimation and explicit form of the Lyapunov function candidate for Eq.(3.11) has also been given in Hong [58]. Consequently, task errors $(e, \dot{e})$ converge for $s=0$ in a finite-time to the origin $(e, \dot{e})=(0,0)$. as follows

In order to fulfil equality constraints Eq.(2.11), a (simple) robust task space control law is proposed

$$
u(t, q, \dot{q}, e, \dot{e})= \begin{cases}-\frac{c}{a} \frac{s}{\|s\|}\left(\mathcal{V}(t, e, \dot{e})+\mathcal{W}(t, q, \dot{q})+c^{\prime}\right) & \text { for } \quad s \neq 0 \\ 0 & \text { otherwise }\end{cases}
$$

where $\mathcal{V}=\left\|\lambda_{0} e^{\alpha_{1}}+\lambda_{l}(\dot{e})^{\alpha_{2}}-\ddot{p}_{d}\right\| ; c, c^{\prime}$ are positive constant gains to be specified further on. Consequently, vector $v$ can be found from the following equation with $u$ given by Eq.(3.12)

$$
v=J^{T}(q, \hat{X}) u(t, q, \dot{q}, e, \dot{e})
$$

Let us observe that control law Eq.(3.12), Eq.(3.13) requires on-line measurements of quantities $q, \dot{q}, e$ and $\dot{e}$, respectively which are assumed for a moment to be available. The aim is to provide conditions on controller gains $\lambda_{0}, \lambda_{l}, c$ and $c^{\prime}$, which guarantee fulfilment of equalities Eq.(2.11). Applying the Lyapunov stability theory, we now derive the following result.

Theorem 1. If $\hat{J}$ fulfils inequality Eq.(3.3) along desired end-effector trajectory $p_{d}, c>\frac{1}{1-\delta}, c^{\prime}>0$ and $\lambda_{0}$, $\lambda_{1}>0$, then control scheme Eqs (3.12)-(3.13) guarantees stable convergence in a finite time of the task space tracking errors $(e, \dot{e})$ to the origin $(e, \dot{e})=(0,0)$.

Proof. Consider the following Lyapunov function candidate

$$
V=\frac{1}{2}\langle s, s\rangle
$$

Differentiating Eq.(3.14) with respect to time and taking into account definition Eq.(3.10) results in the following expression 


$$
\dot{V}=\left\langle s, \ddot{e}+\lambda_{0} e^{\alpha_{1}}+\lambda_{1}(\dot{e})^{\alpha_{2}}\right\rangle .
$$

Inserting the right hand side of Eq.(3.8) into Eq.(3.15) results in

$$
\dot{V}=\left\langle s, J M^{-1} \hat{J}^{T} u\right\rangle+\left\langle s, \lambda_{0} e^{\alpha_{1}}+\lambda_{l}(\dot{e})^{\alpha_{2}}-\ddot{p}_{d}\right\rangle+\langle s, Q\rangle
$$

Substituting $u$ from Eq.(3.16) for the right-hand side of Eq.(3.12) gives the following equality

$$
\dot{V}=-\left\langle s, J M^{-1} \hat{J}^{T} \frac{c}{a} \frac{s}{\|s\|}\left(\mathcal{V}+\mathcal{W}+c^{\prime}\right)\right\rangle+\left\langle s, \lambda_{0} e^{\alpha_{1}}+\lambda_{l}(\dot{e})^{\alpha_{2}}-\ddot{p}_{d}\right\rangle+\langle s, Q\rangle .
$$

Let us transform the first term of the right hand side of Eq.(3.17) into a convenient form

$$
-\left\langle s, J M^{-1} \hat{J}^{T} \frac{c}{a} \frac{s}{\|s\|}\left(\mathcal{V}+\mathcal{W}+c^{\prime}\right)\right\rangle=-\frac{c}{a\|s\|}\left(\mathcal{V}+\mathcal{W}+c^{\prime}\right)\left[\left\langle s, \hat{J} M^{-1} \hat{J}^{T} s\right\rangle+\left\langle s,(J-\hat{J}) M^{-1} \hat{J}^{T} s\right\rangle\right] .
$$

Based on the assumption regarding the rank of matrix $\hat{J}$, expression Eq.(3.3) and using obvious inequality $\|\hat{J}\|_{F} \leq\|J\|_{F}$, we obtain, after simple algebra, the following estimation of the first term of $\dot{V}$ from Eq.(3.17)

$$
\begin{aligned}
& -\left\langle s, J M^{-1} \hat{J}^{T} \frac{c}{a} \frac{s}{\|s\|}\left(\mathcal{V}+\mathcal{W}+c^{\prime}\right)\right\rangle \leq-c\|s\|\left(\mathcal{V}+\mathcal{W}+c^{\prime}\right)+ \\
& +\frac{c}{a}\|s\|\|J-\hat{J}\|_{F} \Lambda_{\max }\|J\|_{F}\left(\mathcal{V}+\mathcal{W}+c^{\prime}\right) .
\end{aligned}
$$

From Eq.(3.4), it follows that

$$
-\left\langle s, J M^{-1} \hat{J}^{T} \frac{c}{a} \frac{s}{\|s\|}\left(\mathcal{V}+\mathcal{W}+c^{\prime}\right)\right\rangle \leq-\|s\|\left(\mathcal{V}+\mathcal{W}+c^{\prime}\right)(1-\delta) c
$$

Let us estimate the sum of the last two terms of $\dot{V}$ from Eq.(3.17). Applying assumption regarding $c^{\prime}$ from Theorem 1, we obtain after simple algebra that

$$
\left\langle s, \lambda_{0} e^{\alpha_{1}}+\lambda_{l}(\dot{e})^{\alpha_{2}}-\ddot{p}_{d}\right\rangle+\langle s, Q\rangle \leq\|s\|\left(\mathcal{V}+\mathcal{W}+c^{\prime}\right) .
$$

Consequently, $\dot{V}$ can be upper estimated as follows

$$
\dot{V} \leq\|s\|\left(\mathcal{V}+\mathcal{W}+c^{\prime}\right)[-c(1-\delta)+1] .
$$

Based on the assumption regarding $c$ and $c^{\prime}$ from Theorem 1, we finally obtain that 


$$
\dot{V} \leq\|s\|\left(\mathcal{V}+\mathcal{W}+c^{\prime}\right)[-c(1-\delta)+1] \leq c^{\prime}[-c(1-\delta)+1]\|s\| .
$$

Since $-c(1-\delta)+1<0$, inequality Eq.(3.22) proves that TSM $s=0$ is attainable in a finite time $T^{\prime}$ less or equal to $\frac{\sqrt{2 V(0)}}{c^{\prime}|-c(1-\delta)+1|}$. Consequently, from Lemma 1 , it follows that the origin $(e, \dot{e})=(0,0)$ is attainable in a finite time $T \geq T^{\prime}$.

A few remarks may be made regarding the controller Eqs (3.12)-(3.13) and Theorem 1.

Remark 1. Observe that control law given by Eqs (3.12)-(3.13) is a feed-back adjustable function equal to $\mathcal{V}+\mathcal{W}+c^{\prime}$. The control laws known from the literature (see e.g. Bartolini et al. [59], Bartolini et al. [60], Bartolini et al. [61], Ferrara and Capisani [62], Spong and Vidyasagar [49], Siciliano et al. [4]) require boundedness of $\dot{q}$ which implies large controller gains to cope with the uncertainty over the whole operation region.

Remark 2. It is also worth to notice that our feed-back adjustable amplitude term $\frac{c}{a}\left(\mathcal{V}+\mathcal{W}+c^{\prime}\right)$ makes it possible to cope with globally unbounded uncertainties. In general, in that case, only local uncertainty suppression is available in the literature for redundant systems.

Remark 3. Non-singular sliding variable $s$ defined by Eq.(3.10) has a nice property for a typical regulation task $\dot{e}(0)=0$ which implies $s(0)=0$. If this is the case, from Eq.(3.22), $s(0)=0$ and Eq.(3.14), it follows that $V(t)=0$ and consequently $s(t)=0($ TSM $s=0$ is attained at $t=0)$.

Remark 4. Let us note that expressions Eqs (3.12)-(3.13) present also a transposed Jacobian controller. However, works Kelly [36], Cheah [37], Cheah et al. [38], Moosavian and Papadopoulos [39] have shown stability of the performance for the set-point control problems. On the other hand, Theorem 1 provides stability analysis for the trajectory tracking problems. Moreover, transposed Jacobian controller Eqs (3.12)(3.13) is able to attain the stable equilibrium $(e, \dot{e})=(0,0)$ in a finite time. Due to involving the sliding mode term in $u$, controller Eqs (3.12)-(3.13) is also robust against uncertainties of both kinematic and dynamic equations as well as external unbounded disturbances.

Remark 5. In controller Eqs (3.12)-(3.13), the term $\frac{s}{\|s\|}$ will cause undesirable chattering effect in a small neighbourhood of $s=0$ which results in torque oscillations and consequently in robot vibrations. In order to eliminate the chattering and consequently reduce robot vibrations, a boundary layer control law may be used in place of $u$ given by Eq.(3.12) of the following form

$$
u(t, q, \dot{q}, e, \dot{e})=\left\{\begin{array}{l}
-\frac{c}{a} \frac{s}{\|s\|}\left(\mathcal{V}+\mathcal{W}+c^{\prime}\right) \text { for }\|s\| \geq \epsilon, \\
-\frac{c}{a} \frac{s}{\epsilon}\left(\mathcal{V}+\mathcal{W}+c^{\prime}\right) \text { otherwise }
\end{array}\right.
$$

where $\epsilon$ is a user specified arbitrarily small positive real number. Substituting $u$ in Eq.(3.16) for the righthand side of Eq.(3.23), we have after simple algebra that 


$$
\begin{aligned}
& \left\langle s, J M^{-1} \hat{J}^{T} u\right\rangle+\left\langle s, \lambda_{0} e^{\alpha_{1}}+\lambda_{l}(\dot{e})^{\alpha_{2}}-\ddot{p}_{d}\right\rangle+ \\
& +\langle s, Q\rangle \leq\|s\|\left(\mathcal{V}+\mathcal{W}+c^{\prime}\right)\left[-\frac{c\|s\|}{\epsilon}(1-\delta)+1\right] .
\end{aligned}
$$

Consequently, $\left\langle s, J M^{-1} \hat{J}^{T} u\right\rangle+\left\langle s, \lambda_{0} e^{\alpha_{1}}+\lambda_{l}(\dot{e})^{\alpha_{2}}-\ddot{p}_{d}\right\rangle+\langle s, Q\rangle \leq 0$ for $\|s=s(t, \epsilon)\| \geq \frac{\epsilon}{c(1-\delta)}$.

Since $s(t, \epsilon)$ is bounded for $t \geq T^{\prime}$ and continuously differentiable mapping with respect to time, we conclude that $\dot{s}(t, \epsilon)$ is bounded too, i.e., $\|\dot{s}(t, \epsilon)\| \leq \epsilon^{\prime}$ in an arbitrarily large but finite and closed time interval; $\epsilon^{\prime}<\infty$. Differentiating equation Eq.(3.10) with respect to time results in the following expression

$$
\ddot{e}=\dot{s}(t, \epsilon)-\lambda_{0} e^{\alpha_{1}}-\lambda_{l}(\dot{e})^{\alpha_{2}}
$$

for $\epsilon>0$ and $t \geq 0$. Moreover, from the proof of Theorem 1, it follows that $\ddot{e}=-\lambda_{0} e^{\alpha_{1}}-\lambda_{l}(\dot{e})^{\alpha_{2}}$ for $t \geq T^{\prime}$ and $\epsilon=0$. Introducing auxiliary Lyapunov function candidate $V_{a}=\frac{\lambda_{0}}{1+\alpha_{1}} e^{1+\alpha_{1}}+\frac{1}{2}\langle\dot{e}, \dot{e}\rangle$ then taking its derivative and using Eq.(3.24), we obtain after simple algebra that $\dot{V}_{a} \leq\|\dot{e}\|\left(\|\dot{s}\|-\min _{i}\left\{\lambda_{1, i}\right\}\|\dot{e}\|^{\alpha_{2}}\right)$. Consequently, $\dot{V}_{a} \leq 0$ for $\|\dot{e}\| \geq\left(\frac{\epsilon^{\prime}}{\min _{i}\left\{\lambda_{1, i}\right\}}\right)^{\frac{1}{\alpha_{2}}}$ which means boundedness of $\|\dot{e}\|$. Furthermore, $\frac{\lambda_{0}}{1+\alpha_{1}} e^{1+\alpha_{1}} \leq \frac{\lambda_{0}}{1+\alpha_{1}} e(0)^{1+\alpha_{1}}+\frac{1}{2}\langle\dot{e}(0), \dot{e}(0)\rangle$ which implies boundedness of $e$. Moreover, the following inequality holds true for each $e, \dot{e}, \quad t^{\prime} \in\left[T, T^{\prime \prime}\right], \quad T<T^{\prime \prime}<\infty$ and $\epsilon>0$ : $\left\|\int_{T}^{t^{\prime}}\left(\dot{s}(t, \epsilon)-\lambda_{0} e^{\alpha_{1}}-\lambda_{l}(\dot{e})^{\alpha_{2}}\right) d t-\int_{T}^{t^{\prime}}\left(\left.\dot{s}(t, \epsilon)\right|_{\epsilon=0}-\lambda_{0} e^{\alpha_{1}}-\lambda_{l}(\dot{e})^{\alpha_{2}}\right) d t\right\| \leq \frac{2 \epsilon}{c(1-\delta)}, \quad$ where $\left.\quad \dot{s}(t, \epsilon)\right|_{\epsilon=0}=0$, which means uniform convergence with respect to $t^{\prime}$ of integral $\int_{T}^{t^{\prime}}\left(\dot{s}(t, \epsilon)-\lambda_{0} e^{\alpha_{1}}-\lambda_{1}(\dot{e})^{\alpha_{2}}\right) d t$ to $\int_{T}^{t^{\prime}}\left(\left.\dot{s}(t, \epsilon)\right|_{\epsilon=0}-\lambda_{0} e^{\alpha_{1}}-\lambda_{I}(\dot{e})^{\alpha_{2}}\right) d t$ as $\epsilon \rightarrow 0$. Finally, from Filippov [63] (Theorem 7), it follows that task error $e=e(t, \epsilon)$ as being the solution of control problem Eq.(3.13) and Eq.(3.23), converges uniformly to $\left.e(t, \epsilon)\right|_{\epsilon=0}=0$, i.e., $(\forall \gamma>0)(\exists \epsilon>0)(\|e(t, \epsilon)\| \leq \gamma)$ for $t \geq T$. Consequently, control law Eq.(3.13) and Eq.(3.23) guarantees that the norm bounds on $e(t, \epsilon), \dot{e}(t, \epsilon)$ can be made arbitrarily small without increase of controller gains.

Remark 6. Let us note that the performance improvement of controller Eq.(3.13) is achieved by an increase of control contribution $\mathcal{V}(t, e, \dot{e})$ in Eq.(3.12) for small tracking errors, because for $0<\alpha_{1}<1$ and $\left|e_{i}(t)\right|<1$ we have $\left|e_{i}(t)\right|^{\alpha_{1}}>\left|e_{i}(t)\right|$. Also, when the tracking error is large (especially at the beginning of the control process), the terminal sliding mode controller Eq.(3.13) gives smaller control effort than that resulting from a linear sliding mode since $\left|e_{i}(t)\right|^{\alpha_{1}}<\left|e_{i}(t)\right|$ for $\left|e_{i}(t)\right|>1$. 
Remark 7. Observe that term $\left\langle s, J M^{-1} \hat{J} u\right\rangle$ in Eq.(3.16) can be transformed after simple algebra as follows $\left\langle s, J M^{-1} \hat{J} u\right\rangle=-\frac{c}{a\|s\|}\left(\mathcal{V}+\mathcal{W}+c^{\prime}\right)\left[\left\langle s, \hat{J} M^{-1} \hat{J}^{T} s\right\rangle+\left\langle s,(J-\hat{J}) M^{-1} \hat{J}^{T} s\right\rangle\right]$. Let $\hat{J} \quad$ be singular at manipulator configuration $q^{\prime}$ and $0 \neq s \notin \operatorname{ker}\left(J^{T}\left(q^{\prime}, \hat{X}\right)\right)$. Hence, for sufficiently large $c$, term $\left\langle s, J M^{-1} \hat{J} u\right\rangle$ can take arbitrarily large negative values which implies negative value of time derivative $\dot{V}$. Consequently, controller Eqs (3.12)-(3.13) or Eq.(3.13), Eq.(3.23) is able to generate manipulator motions which can pass through singular manifold $\left\{q^{\prime}: \operatorname{det}\left(J\left(q^{\prime}, \hat{X}\right)\right)=0\right\}$.

Remark 8. Let us note that if the conventional trajectory tracking is only taken into account (without objective function $\mathcal{A}$ ) in the control problem then the robotic manipulator becomes strictly redundant, i.e., $n>m$. In such a case by defining the task space TSM $s^{e}$ as $s^{e}=e^{e}+\int_{0}^{t}\left(\lambda_{0}\left(e^{e}\right)^{\alpha_{1}}+\lambda_{1}\left(e^{e}\right)^{\alpha_{2}}\right) d \tau$, controller Eqs (3.12)-(3.13) may be simplified to the following form

$$
v=\hat{\jmath}^{T} u^{e}\left(t, q, \dot{q}, e^{e}, \dot{e}^{e}\right)
$$

where $\hat{\jmath}=\jmath(q, \hat{X})$ and

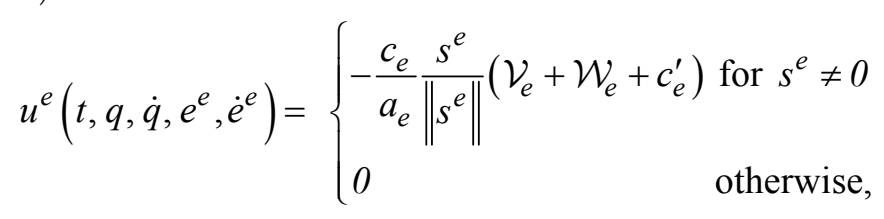

$\mathcal{V}_{e}=\left\|\lambda_{0}\left(e^{e}\right)^{\alpha_{1}}+\lambda_{I}\left(e^{e}\right)^{\alpha_{2}}-\ddot{p}_{d}^{e}\right\| ; \quad \mathcal{W}_{e}=\|\|_{F} \cdot \Lambda_{\max } \cdot \mathcal{E}+\left\|\frac{\partial \jmath}{\partial q}\right\|_{F} \cdot\|\dot{q}\|^{2} ; \quad c_{e}>\frac{1}{1-\delta}, \quad c_{e}^{\prime}>0 ; \quad a_{e} \quad$ denotes estimation of the minimal eigenvalue of matrix $\hat{\jmath} M^{-1} \hat{\jmath}^{T}$ along desired trajectory $p_{d} ;\|\jmath\|_{F} \leq c_{3} ;\left\|\frac{\partial \jmath}{\partial q}\right\|_{F} \leq c_{5}$.

In most cases, real robotic manipulators are equipped with encoders which measure only joint positions and/or task errors. Hence, reconstruction or estimation of joint velocity and task space error velocity is required to apply controller Eqs (3.12)-(3.13) or Eq.(3.13), Eq.(3.23). Application of Luenberger-style observers Canudas et al. [64], El Beheiry [65], high-gain observers Khalil and Praly [66], Ball and Khalil [67], model-free observers De Luca et al. [68], Hsiao and Weng [69] or a class of observers based on the slidingmode algorithms Davila et al. [70] seems to be an efficient approach to reconstruct both $\dot{q}$ and $\dot{e}$. Although all the aforementioned observers are able to reliably reconstruct manipulator state (both joint velocity and task space error velocity) based on position measurement $q$ and task error $e$, there appears a difficulty to combine our control law and an observer from Canudas et al. [64], El Beheiry et al. [65], Khalil and Praly [66], Ball and Khalil [67], De Luca et al. [68], Hsiao and Weng [69]. In order to make such combination possible, observers proposed in works Canudas et al. [64], El Beheiry et al. [65], Khalil and Praly [66], Ball and Khalil [67], De Luca et al. [68], Hsiao and Weng [69] have to satisfy the so-called separation principle Atasi and Khalil [71] which implies both the continuity of the controllers from Canudas et al. [64], El Beheiry et al. [65], Khalil and Praly [66], Ball and Khalil [67], De Luca et al. [68], Hsiao and Weng [69] 
with the fully available state and asymptotic stability of the closed-loop system under the continuous state feed-back controllers. Let us observe that our control law Eqs (3.12)-(3.13) is discontinuous and Eq.(3.13), Eq.(3.23) is not asymptotically stable what prevents an application of the state observers from Canudas et al. [64], El Beheiry et al. [65], Khalil and Praly [66], Ball and Khalil [67], De Luca et al. [68], Hsiao and Weng [69]. Although the observer offered in Davila et al. [70] fulfils the separation principle, our controller handles unbounded uncertainties (in kinematics, dynamics and disturbances) and does not require boundedness of $\dot{q}$ and $\dot{e}$, respectively. A computationally efficient approach based on the uniform robust exact finite-time differentiation has been recently proposed in works Levant and Livne [72], Levant [73] to numerically find derivatives of absolutely continuous functions. The separation principle is trivially fulfilled for differentiators (model-free observers) from Levant and Livne [72], Levant [73]. Assuming that position $q=q(t)$ and task error $e=e(t)$ are known (measurable), one can exactly reconstruct both joint velocity $\dot{q}(t)$ and task error velocity $\dot{e}(t)$ (by neglecting the measurement noise of a device) after finite-times of transient processes, say $T_{q}^{\prime}, T_{e}^{\prime}>0$, respectively. The first-order uniform robust exact differentiators (model-free observers) take in our case the following forms

$$
\begin{aligned}
& \dot{y}_{0}=y_{1}-\hat{\lambda}_{l}^{q} L_{q}(t)^{1 / 2}\left|y_{0}-q\right|^{1 / 2} \operatorname{sign}\left(y_{0}-q\right), \\
& \dot{y}_{1}=-\hat{\lambda}_{0}^{q} L_{q}(t) \operatorname{sign}\left(y_{0}-q\right),
\end{aligned}
$$

and

$$
\begin{aligned}
& \dot{z}_{0}=z_{1}-\hat{\lambda}_{1}^{e} L_{e}(t)^{1 / 2}\left|z_{0}-e\right|^{1 / 2} \operatorname{sign}\left(z_{0}-e\right), \\
& \dot{z}_{1}=-\hat{\lambda}_{0}^{e} L_{e}(t) \operatorname{sign}\left(z_{0}-e\right),
\end{aligned}
$$

where $\hat{\lambda}_{0}^{q}, \hat{\lambda}_{1}^{q}, \hat{\lambda}_{0}^{e}, \hat{\lambda}_{1}^{e}$, are positive constants. $y_{1}, z_{1}$ denote the outputs of differentiators Eqs (3.27)-(3.28) exactly reconstructing joint velocity $\dot{q}(t)$ and task error velocity $\dot{e}(t)$, i.e., $\dot{q}(t)=y_{l}(t), \dot{e}(t)=z_{l}(t)$ for $t \geq \max \left\{T_{q}^{\prime}, T_{e}^{\prime}\right\} \cdot y_{0}, z_{0}$ stand for estimations of $q$ and $e$, respectively. By substituting $\dot{q}$ and $\dot{e}$ in Eq.(3.12) or Eq.(3.23) for their corresponding estimates $y_{1}$ and $z_{1}$ from Eqs (3.27)-(3.28), we obtain the following trajectory tracking controller

$$
v=\hat{J}^{T} u\left(t, q, y_{1}, e, z_{1}\right),
$$

with

$$
u\left(t, q, y_{1}, e, z_{l}\right)= \begin{cases}-\frac{c}{a} \frac{s}{\|s\|}\left(\mathcal{V}+\mathcal{W}+c^{\prime}\right) & \text { for }\|s\| \geq \epsilon \\ -\frac{c}{a} \frac{s}{\epsilon}\left(\mathcal{V}+\mathcal{W}+c^{\prime}\right) & \text { otherwise }\end{cases}
$$

which requires only the measurements of joint position $q$ and task error $e . L_{q}(t), L_{e}(t)$ stand for positive continuous functions which take the forms $L_{q}(t)=\Lambda_{\max }(\|v\|+\mathcal{E})$ and $L_{e}(t)=\|J\|_{F} \cdot L_{q}(t)+\left\|\frac{\partial J}{\partial q}\right\|_{F} \cdot\left\|y_{1}\right\|^{2}+\left\|\ddot{p}_{d}\right\|$, 
respectively. $L_{q}(t), L_{e}(t)$ represent physically upper estimations of the norms of $\ddot{q}, \ddot{e}$ (manipulator joint acceleration and task error acceleration), respectively. In a general case, if measured joint position $q=q(t)$ and task error $e=e(t)$, obtained from encoders, are additionally contaminated by a measurement noise $\eta(t)$, i.e., $q(t)=q_{0}(t)+\eta(t), e(t)=e_{0}(t)+\eta(t)$, where $\|\eta\| \leq \rho \cdot \min \left\{L_{q}(t), L_{e}(t)\right\} ; \rho$ denotes a normalised noise magnitude (practically $\left.\rho \in\left[10^{-4}, 10^{-3}\right]\right) ; q_{0}(t), \dot{q}_{0}(t), e_{0}(t), \dot{e}_{0}(t)$ stand for unknown true (noise-free) joint position, velocity, task error and task error velocity, respectively, then observers Eq.(3.27), Eq.(3.28) should also be applied to estimate quantities $e$ and $\dot{e}$. Notice from equations Eq.(3.27), Eq.(3.28) that $\left\|q_{0}(t)-y_{0}(t)\right\| \leq L_{q}(t) O(\rho), \quad\left\|\dot{q}_{0}(t)-y_{1}(t)\right\| \leq L_{q}(t) O\left(\rho^{1 / 2}\right) \quad$ and $\quad\left\|e_{0}(t)-z_{0}(t)\right\| \leq L_{e}(t) O(\rho)$, $\left\|\dot{e}_{0}(t)-z_{l}(t)\right\| \leq L_{e}(t) O\left(\rho^{1 / 2}\right)$ after a finite transient time Levant and Livne [72], Levant [73]. Moreover, we know from Remark 5. that $\left\|e_{0}\right\| \leq O(\gamma),\left\|\dot{e}_{0}\right\| \leq O(\gamma)$ after a finite transient time too, where $O(\gamma)$ is the same order as $\gamma$. Consequently, for task error norm estimation $\|\hat{e}\|=\left\|z_{0}\right\|$ and $\|\hat{e}\|=\left\|z_{1}\right\|$, we obtain after simple algebra the following upper estimates for chattering-free torque-reduced controller Eqs (3.29)-(3.30) subject to the measurement noise: $\|\hat{e}\| \leq L_{e}(t) O(\rho)+O(\gamma) ;\|\hat{\hat{e}}\| \leq L_{e}(t) O\left(\rho^{1 / 2}\right)+O(\gamma)$.

\section{Numerical examples}

This section demonstrates the performance of the controllers given by expressions Eqs (3.12)-(3.13); Eq.(3.13), Eq.(3.23); Eqs (3.25)-(3.26) and Eqs (3.29)-(3.30) on a selected robotic manipulator task. Moreover, a numerical comparison of our control schemes to other well known controllers is also drawn. For this purpose, we utilise a dynamic three-joint direct-drive arm $(n=3)$ of a SCARA-type robotic manipulator operating in the two-dimensional task space $(m=2)$ whose kinematic scheme is shown in Fig.1, where $X=\left(X_{1}, X_{2}, X_{3}\right)^{T}$ stands for the ordered set of link lengths.

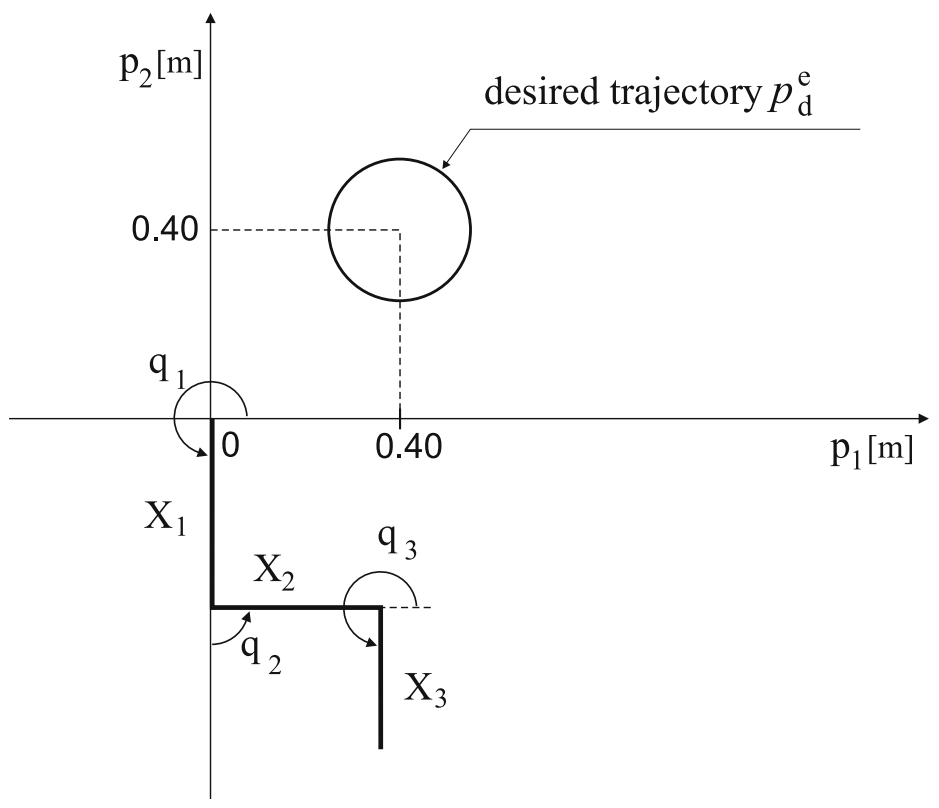

Fig.1. A kinematic scheme of the manipulator and the task to be accomplished. 
In all numerical simulations, the SI units are used. The (nominal) link lengths are assumed to be equal to $X_{n}=(0.40 .360 .3)^{T}$. The estimation term $\hat{X}$ takes in all the simulations the form as follows $\hat{X}=(0.50 .280 .35)^{T}$. The coefficients of disturbance term $D$ and their estimations are assumed in all the experiments to take the following scalar values: $d_{0}=\bar{d}_{0}=2, d_{1}=\bar{d}_{1}=2, d_{2}=5, \bar{d}_{2}=7, d_{3}=2$, $\bar{d}_{3}=3, d_{4}=0.2$ and $\underline{d}_{4}=0$, respectively. In order to numerically compare our controllers with those known from the literature, we reformulate dynamic equations Eq.(2.1), Eq.(2.12) to a partially linearly parametrizable form as follows Spong and Vidyasagar [49]

$$
M \dot{r}+C r+\Theta\left(e^{e}, \dot{e}^{e}, q, \dot{q}, \mathcal{A}, \dot{\mathcal{A}}\right) Y+d_{2} \operatorname{sign}(\dot{q})+d_{3} \exp \left(-d_{4}\|\dot{q}\|^{2}\right) \operatorname{sign}(\dot{q})=v
$$

where $r=\dot{q}-\jmath^{\#}\left(\dot{p}_{d}-\alpha e^{e}\right)-\left(\mathbb{I}_{n}-\jmath^{\#} \jmath\right) 10 \frac{\partial \mathcal{A}}{\partial q}$ denotes a filtered tracking error signal Zergeroglu et al. [21], Ozbay et al. [22], $\jmath=\jmath\left(q, X_{n}\right) ; \quad \Theta=\Theta\left(e^{e}, \dot{e}{ }^{e}, q, \dot{q}, \mathcal{A}, \dot{\mathcal{A}}\right) \in \mathbb{R}^{n \times d} \quad$ is $\quad$ the regression matrix; $\Theta Y=M \frac{d}{d t}\left(\jmath^{\#}\left(\dot{p}_{d}-\alpha e^{e}\right)+\left(\mathbb{I}_{n}-\jmath^{\#} \jmath\right) 10 \frac{\partial \mathcal{A}}{\partial q}\right)+C\left(\jmath^{\#}\left(\dot{p}_{d}-\alpha e^{e}\right)+\left(\mathbb{I}_{n}-\jmath^{\#} \jmath\right) 10 \frac{\partial \mathcal{A}}{\partial q}\right)+G+d_{0}(q-q(0))+d_{1} \dot{q} ; \quad d$ stands for the minimal number of the dynamic parameters, which are combinations of physical link parameters of individual links and coefficients of continuous linear friction terms; $Y$ is the constant parameters vector (link mass, link inertia, friction coefficients, etc.); $\alpha$ stands for a constant gain coefficient. The components of the dynamic equations of the manipulator from Fig.1 (without disturbance term $D$ ) are as follows Galicki [20]

$$
\begin{aligned}
& M=\left[M_{i j}\right]_{1 \leq i, j \leq 3}, \\
& M_{11}=Y_{1}+2 Y_{4} c 2+Y_{5} c 23+Y_{6} c 3, \\
& M_{12}=Y_{2}+Y_{4} c 2+\frac{Y_{5}}{2} c 23+Y_{6} c 3, \\
& M_{13}=Y_{3}+\frac{Y_{5}}{2} c 23+\frac{Y_{6}}{2} c 3, \\
& M_{21}=M_{12}, \\
& M_{22}=Y_{2}+Y_{6} c 3, \\
& M_{23}=Y_{3}+\frac{Y_{6}}{2} c 3, \\
& M_{31}=M_{13},
\end{aligned}
$$




$$
\begin{aligned}
& M_{32}=M_{23}, \\
& M_{33}=Y_{3}, \\
& C=\left[C_{i j}\right]_{1 \leq i, j \leq 3}, \\
& C_{11}=-\left(Y_{4} s 2+Y_{5} s 12\right) \dot{q}_{2}-\left(Y_{5} s 12+Y_{6} s 12\right) \dot{q}_{3}, \\
& C_{12}=-\left(Y_{4} s 2+Y_{5} s 12\right)\left(\dot{q}_{1}+\dot{q}_{2}\right)-\left(Y_{5} s 12+Y_{6} s 12\right) \dot{q}_{3}, \\
& C_{13}=\left(Y_{5} s 12+Y_{6} s 12\right)\left(-\dot{q}_{1}+\dot{q}_{2}+\dot{q}_{3}\right), \\
& C_{21}=\left(Y_{4} s 2+Y_{5} s 12\right) \dot{q}_{1}+Y_{6} s 3 \dot{q}_{3}, \\
& C_{22}=-\left(Y_{5} s 12+Y_{6} s 12\right) \dot{q}_{3}, \\
& C_{23}=-Y_{6} s 3\left(3 \dot{q}_{1}+\dot{q}_{2}+\dot{q}_{3}\right) \\
& C_{31}=\left(Y_{4} s 2+Y_{5} s 12\right) \dot{q}_{1}-Y_{6} s 3 \dot{q}_{2}, \\
& C_{32}=Y_{6} s 3\left(\dot{q}_{1}+\dot{q}_{2}\right), \\
& C_{33}=0 \text {, } \\
& G=\left(\begin{array}{c}
Y_{7} c 1+Y_{8} c 12+Y_{9} c 123 \\
Y_{8} c 12+Y_{9} c 123 \\
Y_{9} c 123
\end{array}\right),
\end{aligned}
$$

where $\quad c i=\cos \left(q_{i}\right) ; \quad s i=\sin \left(q_{i}\right) ; \quad c i j=\cos \left(q_{i}+q_{j}\right) ; \quad s i j=\sin \left(q_{i}+q_{j}\right) ; \quad c i j k=\cos \left(q_{i}+q_{j}+q_{k}\right)$; $\operatorname{sijk}=\sin \left(q_{i}+q_{j}+q_{k}\right)$. Parameters $Y=\left(Y_{1}, \ldots, Y_{9}, d_{0}, d_{1}\right)^{T}$ (including coefficients of continuous linear friction

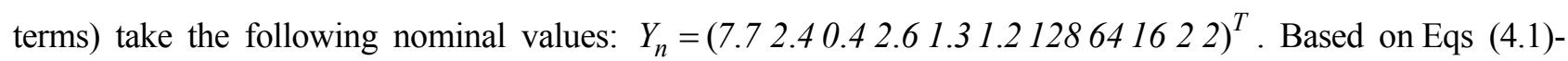
(4.4) and the knowledge of $X_{n}, Y_{n}$, a numerical comparison of our controller Eqs. (3.29)-(3.30) to a well known robust controller from Zergeroglu et al. [21], Ozbay et al. [22] will also be carried out in this section. The control law proposed in Zergeroglu et al. [21], Ozbay et al. [22] is given by the following expression

$$
v=\Theta \hat{Y}+K r-\jmath^{T} e^{e}+v_{R}
$$


where $v_{R}=\frac{r \Delta^{2}}{\|r\| \Delta+\epsilon}$ is an auxiliary robust control term designed to compensate for unknown nonlinear part of disturbances and parameter uncertainties of terms $M, C$ and $G$, respectively; $K$ denotes a gain coefficient, $\hat{Y}$ means the best guess estimates (of the unknown parameters $Y$ ). Let us note that controller Eq.(4.5) requires the full knowledge of kinematic parameters $X$. Moreover, according to Zergeroglu et al. [21], Ozbay et al. [22], the best values for $\Delta$ and $\epsilon$ equal $\Delta=10, \epsilon=0.01$, respectively. The initial configuration and velocity of the manipulator are equal in all the experiments to $q(0)=(-\pi / 2, \pi / 2,0)^{T}, \dot{q}(0)=(0,0,0)^{T}$, respectively. To compare the performance of controllers Eqs (3.12)-(3.13) and Eqs (3.25)-(3.26), our estimates for controllers Eqs (3.12)-(3.13); Eqs (3.25)-(3.26); Eq.(3.13), Eq.(3.23) and Eqs (3.29)-(3.30) are chosen as $a=a_{e}=0.2 ; \Lambda_{\max }=6$. In order to simplify numerical computations, rough conservative estimations of $c_{i}, i=1: 6$ have been assumed. Hence, positive constant coefficients $c_{i}, i=1: 6$ were chosen as follows $c_{1}=10, c_{2}=240, c_{3}=0.07, c_{4}=0.0007, c_{5}=0.00022$ and $c_{6}=0.000022$, respectively.

The task realized by controllers Eqs (3.12)-(3.13); Eqs (3.25)-(3.26); Eq.(3.13), Eq.(3.23); Eqs (3.29)-(3.30) and Eq.(4.5), is to track by the end-effector a circle trajectory, expressed by the following equations (see the solid circle trajectory in Fig.1)

$$
p_{d}^{e}(t)=(0.4+0.15 \cos (t), 0.4+0.15 \sin (t))^{T}
$$

In order to attain the convergence of task errors $e^{e}$ less or equal to $10^{-3}$ in approximately the same time, the following numerical values of gain coefficients for all the controllers are taken: $K=245$, $\alpha=374.5, K_{\mathcal{A}}=0.05, c_{\mathcal{A}}=1, q_{\text {rest }}=q(0), c=c_{e}=1.46, c^{\prime}=c_{e}^{\prime}=0.5, \lambda_{0}=51, \lambda_{1}=46$ and $\alpha_{1}=\frac{3}{5}$, respectively. In order to speed up the convergence process of differentiators Eqs (3.27)-(3.28), we have chosen good initial guesses $y_{l}(0), z_{l}(0)$ in the numerical examples (which imply relations $T_{q}^{\prime} \simeq 0$ and $T_{e}^{\prime} \simeq 0$ ) based on the nominal values of both our kinematic and dynamic models. Consequently, differentiators Eqs (3.27)-(3.28) were run with the following initial values $y_{0}=q(0), y_{1}(0)=\dot{q}(0), \quad z_{0}(0)=e(0)=(0.11-0.80)^{T}$, $z_{l}(0)=\dot{e}(0)=(0-0.150)^{T}$ and parameters $\hat{\lambda}_{0}^{q}=\hat{\lambda}_{0}^{e}=1.1 ; \hat{\lambda}_{l}^{q}=\hat{\lambda}_{l}^{e}=1.4$, respectively. Due to conservative nature of estimates $L_{q}$ and $L_{e}$ in Eqs (3.27)-(3.28), they are assumed for simplicity of computations in all the simulations to be equal to $L_{e}(t)=L_{q}(t)=\Lambda_{\max }(\|v\|+\mathcal{E})$. Let us also observe that filtered tracking error $r$ in Eq.(4.5) plays a role of linear sliding variable with respect to $e^{e}$ whereas its corresponding term $s$ in Eqs (3.29)-(3.30) is a non-linear function of $e^{e}$. In order to better visualise time courses of the task errors for all the controllers, we omit in the simulations an initial approaching phase of the end-effector to desired trajectory $p_{d}^{e}$. Consequently, the trajectory tracking is exhibited for $t \in\left[\begin{array}{ll}5 & 9\end{array}\right]$.

The aim of the first experiment is to both compare the performance of the two proposed controllers Eqs (3.12)-(3.13); Eqs (3.25)-(3.26) and exhibit the role of objective function $\mathcal{A}$ by reducing the torque $v$ in the $L_{2}[1,9]$ norm. The results of the simulations are depicted in Figs 2-6. As is seen from Figs 2, 4, both controllers generate tracking errors $e^{e}$ which are practically equal to zero. Moreover, Fig.5 indicates that controller Eqs (3.12)-(3.13) provides optimal manipulator movements ( $e^{a}$ practically equals 0 ) whereas control law Eqs (3.25)-(3.26) does not generate optimal solution $\left(e^{a} \neq 0\right)$. As is also seen from Figs 3, 6 , 
control scheme Eqs (3.12)-(3.13) results in smaller torque norm $\overline{\|v\|}=389$ (defined in $L_{2}[1,9]$ norm as $\overline{\|v\|}=\frac{1}{2} \int_{1}^{9} \sqrt{\langle v, v\rangle} d t$ ) than that obtained from Eqs (3.25)-(3.26) and equal to $\overline{\|v\|}=611$.

In order to both eliminate the chattering effect and exhibit the role of feed-back amplitude adjustable term $\frac{c}{a}\left(\mathcal{V}+\mathcal{W}+c^{\prime}\right)$ from Eqs (3.29)-(3.30) compared with the corresponding constant term $\Delta$ from Eq.(4.5) in the second experiment, components of dynamic equations Eqs (4.2)-(4.4) together with coefficients of continuous linear friction terms $d_{0}, d_{1}$ are assumed in this experiment to be fully known, i.e., the following equality is now fulfilled: $\hat{Y}=Y_{n}$. Moreover, application of controller Eq.(4.5) requires the full knowledge of the set $X$, i.e., we assume that $X=X_{n}$. The results of computer simulations are presented

in Figs 7-9. As is seen from Figs 7-8, controller Eqs (3.29)-(3.30) generates tracking errors $e^{e}$ and $e^{a}$ which are also practically equal to zero compared with controller Eq.(4.5) using the full knowledge of both robot kinematic and dynamic equations. This fact is a consequence of Remark 6. and linear dependence of $r$ on $e^{e}$.

Moreover, Fig.8 indicates that controller Eqs (3.29)-(3.30) provides practically optimal manipulator movements whereas control law Eq.(4.5) generates only suboptimal solution (controller Eq.(4.5) results in $\left.e^{a} \neq 0\right)$. As is also seen from Fig.9, control scheme Eqs (3.29)-(3.30) results in smaller torque norm $\overline{\|v\|}=184.7$ than that obtained from Eq.(4.5) and equal to $\overline{\|v\|}=202.8$. This fact is a consequence of both torque minimizing role of objective function $\mathcal{A}$ and Remarks 1, 2 explaining the role of the feed-back adjustable amplitude term $\frac{c}{c}\left(\mathcal{W}+\mathcal{W}+c^{\prime}\right)$ which varies over the manipulator operation region with respect to the changing uncertainty bounds. Alternatively, the corresponding amplitude term $\Delta$ from Eq.(4.5) is constant in the whole operation region.

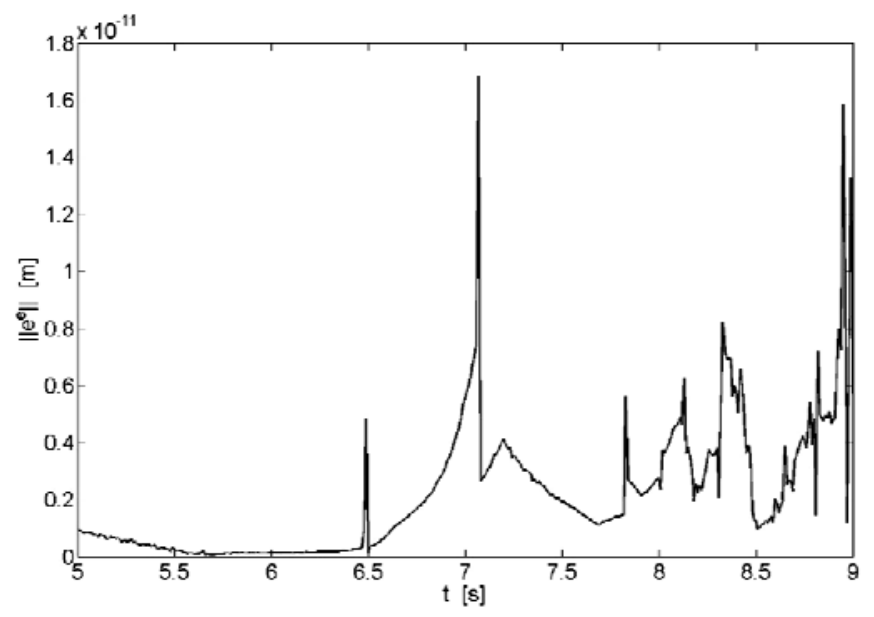

Fig.2. Position errors $e^{e}$ for controller Eqs (3.25)-(3.26) when tracking the trajectory $p_{d}$. 


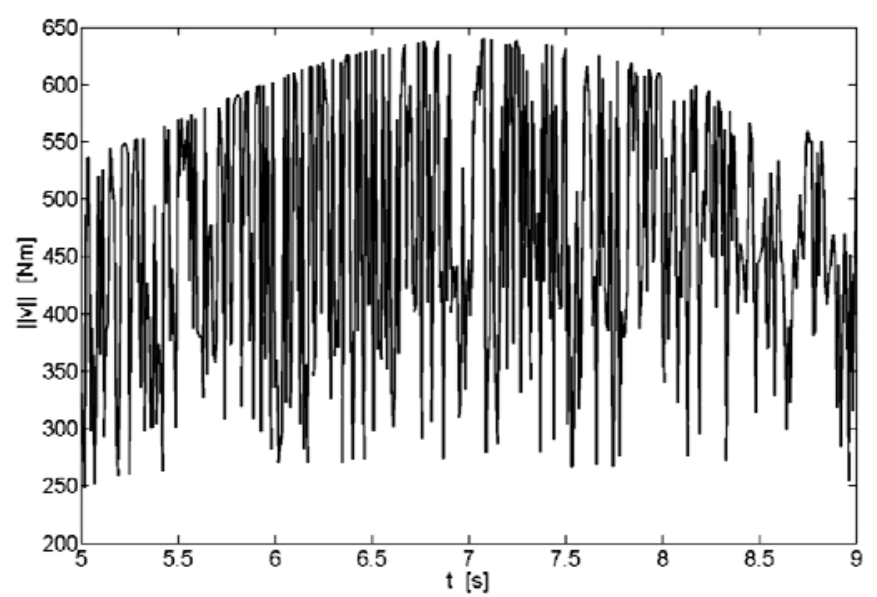

Fig.3. Euclidean norm of $v$ for controller Eqs (3.25)-(3.26) when tracking the trajectory $p_{d}$.

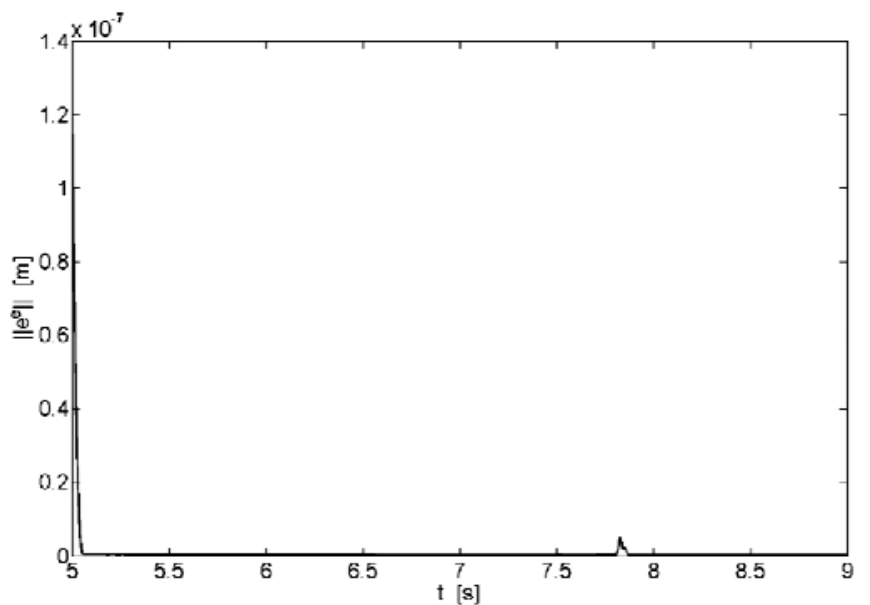

Fig.4. Position errors $e^{e}$ for controller Eqs (3.12)-(3.13) when tracking the trajectory $p_{d}$.

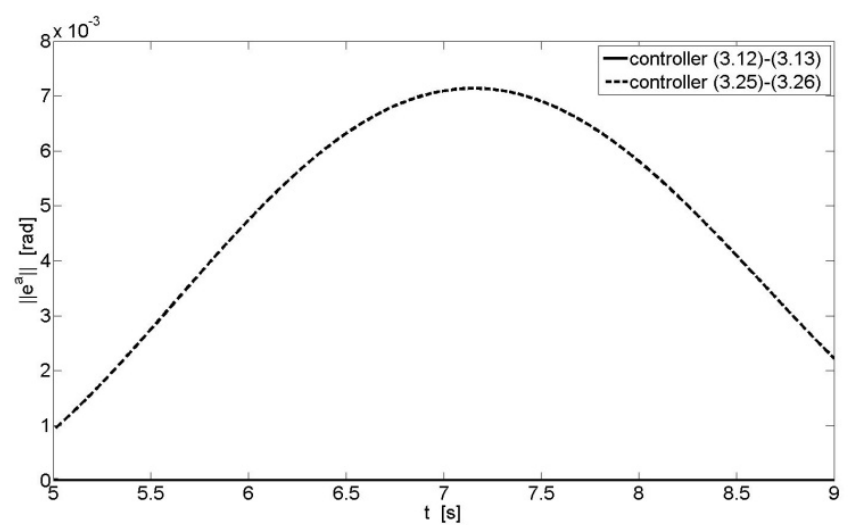

Fig.5. Task errors $e^{a}$ for controllers Eqs (3.12)-(3.13) and Eqs (3.25)-(3.26) when tracking the trajectory $p_{d}$. 


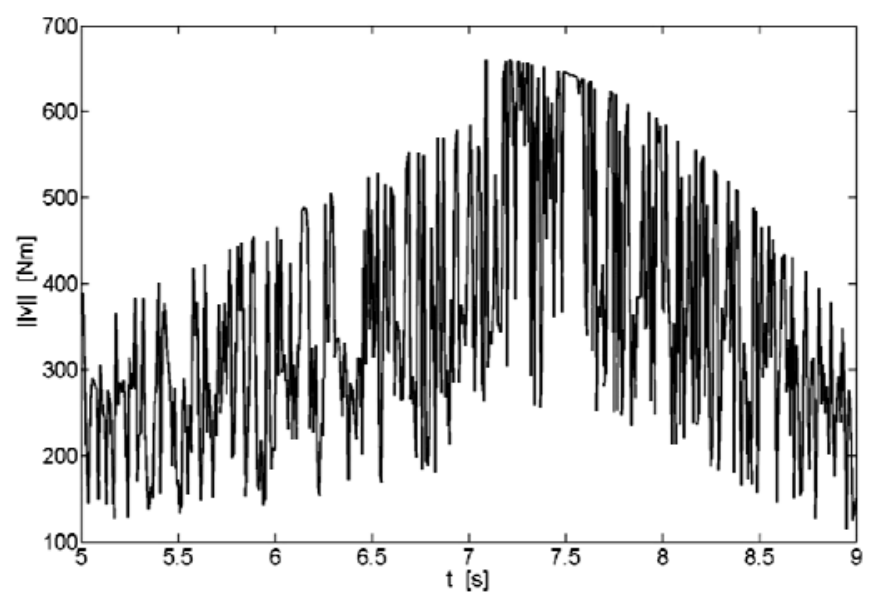

Fig.6. Euclidean norm of $v$ for controller Eqs (3.12)-(3.13) when tracking the trajectory $p_{d}$.

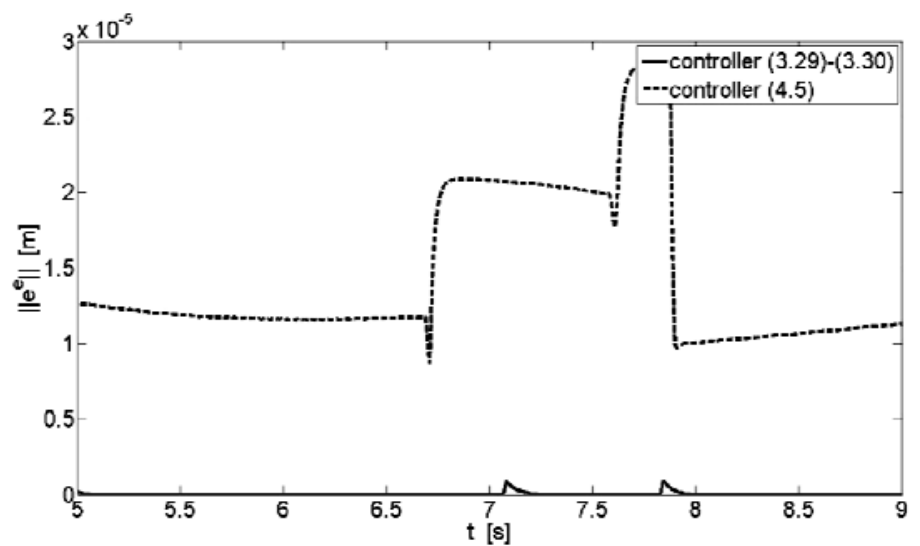

Fig.7. Position errors $e^{e}$ for controllers Eqs (3.29)-(3.30) and (4.5) when tracking the trajectory $p_{d}$.

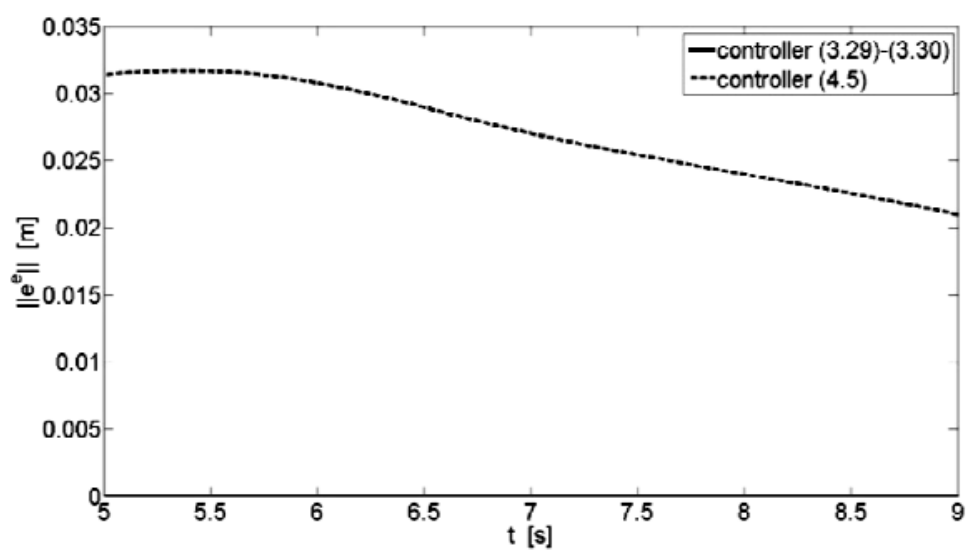

Fig.8. Task errors $e^{a}$ for controllers Eqs (3.29)-(3.30) and (4.5) when tracking the trajectory $p_{d}$. 


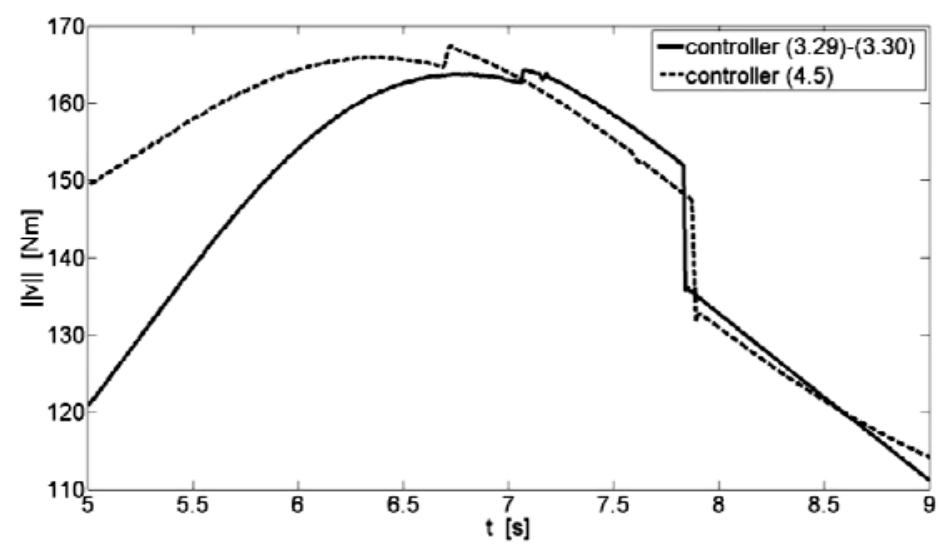

Fig.9. Euclidean norm of $v$ for controllers Eqs (3.29)-(3.30) and (4.5) when tracking the trajectory $p_{d}$.

In the third experiment, both measured position $q$ and task error $e$ have additionally been contaminated by a measurement noise of a Brownian motion of the form $d \eta(t)=10^{-3} \sqrt{t} X(t) d t$ for $t \in[0,9]$; $X(t) \sim N(0,1)$. The results of simulations are given in Figs 10-11 which indicate a good performance of controller Eqs (3.29)-(3.30) subject to measurement noise. The peak of $\|\hat{e}\|$ for $t \simeq 7.2$ in Fig.10 is a result of the Coulomb and Stribeck discontinuity friction terms with respect to $\dot{q}$ depicted in Fig.11.

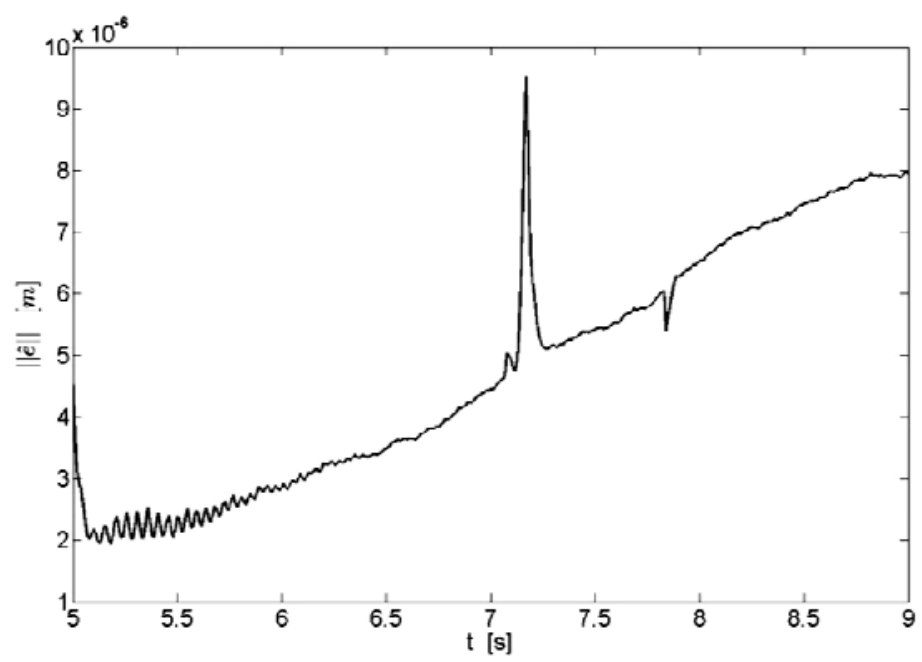

Fig.10. Euclidean norm of estimated task errors $\hat{e}$ for controller Eqs (3.29)-(3.30) with measurement noise. 


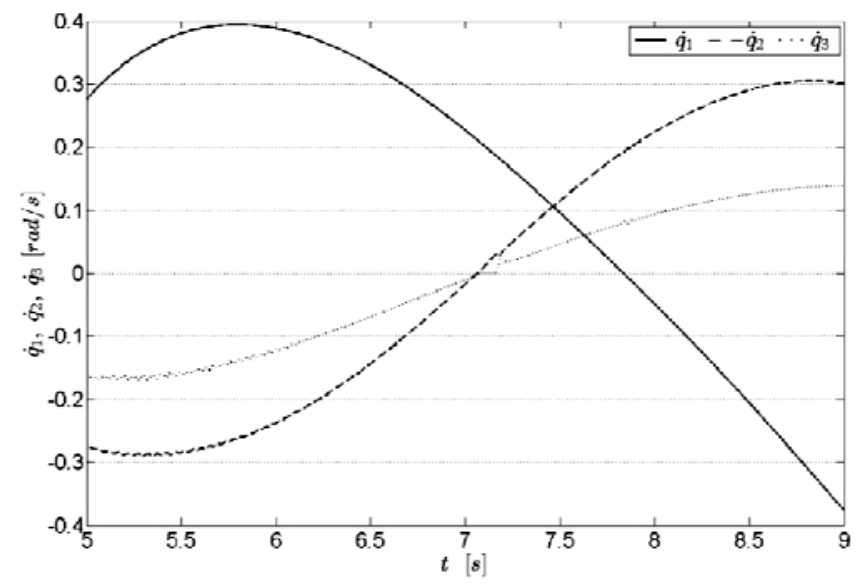

Fig.11. Joint velocities $\dot{q}$ for controller Eqs (3.29)-(3.30) with measurement noise.

\section{Conclusions}

A new class of task space TSM controllers with the finite-time convergence property of the desired trajectory tracking by $n$-DoF redundant manipulator whose end-effector operates in the $m$-dimensional task space, has been proposed in this paper. Moreover, a novel non-singular TSM manifold was incorporated into control scheme. The main feature of the control law proposed is both the elimination of the manipulator Jacobian inverse or pseudo-inverse from the end-effector trajectory tracking and copying with the unbounded disturbances. Instead, estimate of the Jacobian transpose matrix has been used. Applying the Lyapunov stability theory, control strategy Eqs (3.12)-(3.13) is shown to be finite-time stable by fulfilment of practically reasonable assumptions. In order to eliminate undesirable chattering effect, a boundary layer control was proposed guaranteeing arbitrarily small bounds on the tracking errors without increase of the controller gains. Numerical simulations have shown that controller Eqs (3.29)-(3.30) is superior to a well known robust control scheme Eq.(4.5) in accuracy of the trajectory tracking. Although our control technique needs knowledge extracted from the system equations of the robot, the approach is able to handle uncertainty (in kinematics, dynamics and disturbance) occurring in the system.

\section{Nomenclature}

$$
\begin{aligned}
\mathcal{A} & - \text { an objective function to be minimized } \\
C(q, \dot{q}) \dot{q} & - \text { the } n \text {-dimensional vector representing centrifugal and Coriolis forces } \\
D(t, q, \dot{q}) & - \text { the } n \text {-dimensional external disturbance signal } \\
e=\left(e_{1}, \ldots, e_{n}\right)^{T} & \text { - the task tracking error } \\
G(q) & - \text { the } n \text {-dimensional vector of generalized gravity forces } \\
J=\frac{\partial f}{\partial q} & - \text { the } n \times n \text { extended Jacobian matrix } \\
\hat{J}=J(q, \hat{X}) & - \text { estimation of the uncertain Jacobian matrix } \\
k & - \text { the number of kinematic parameters } \\
M(q) & - \text { the } n \times n \text { positive definite, symmetric inertia matrix } \\
\mathcal{N} & - \text { the }(n-m) \times n \text { orthogonal complementary matrix to } J(q, X) \\
n & - \text { the number of kinematic pairs of the V-th class }
\end{aligned}
$$




$$
\begin{aligned}
p=\left(p_{e}^{T} p_{a}^{T}\right)^{T} & - \text { extended task coordinates } \\
p_{a}=\left(p_{a, 1}, \ldots, p_{a, n-m}\right)^{T} & - \text { additional user-specified useful task coordinates } \\
p_{e}=\left(p_{e, 1}, \ldots, p_{e, m}\right)^{T} & - \text { the end-effector coordinates } \\
p_{d}^{e}(t) \in \mathbb{R}^{m} & - \text { a desired end-effector trajectory } \\
p_{d}^{e}(\cdot) \in C^{2} & - \text { desired user specified trajectory } \\
s=\left(s_{1}, \ldots, s_{n}\right)^{T} \in \mathbb{R}^{n} & - \text { a task space sliding vector variable } \\
u \in \mathbb{R}^{n} & - \text { a new control to be determined } \\
q(0) & - \text { initial posture of the manipulator } \\
q=\left(q_{1}, \ldots, q_{n}\right)^{T} & - \text { generalized (joint) coordinates } \\
\dot{q}, \ddot{q} & - \text { velocity and acceleration, respectively expressed in generalized coordinates } \\
X=\left(X_{1}, \ldots, X_{k}\right)^{T} & - \text { an ordered set of kinematic parameters such as link lengths and/or joint offsets } \\
\alpha_{0}(\cdot) & - \text { the time dependent known non-negative locally bounded Lebesgue measurable function } \\
\epsilon & - \text { a user specified arbitrarily small positive real number } \\
\eta(t) & - \text { a measurement noise } \\
v=\left(v_{1}, \ldots, v_{n}\right)^{T} & - \text { the } n \text {-dimensional vector of controls (torques/forces) } \\
0 \leq T & - \text { a finite-time of convergence of } f(q) \text { to } p_{d} \\
\mathbb{I}_{n} & - \text { the } n \times n \text { identity matrix }
\end{aligned}
$$

\section{References}

[1] Khatib O. (1987): A unified approach for motion and force control of robot manipulators. - EEE Journal on Robotics and Automation, vol.3, No.1, pp.43-53.

[2] Hsu P., Hauser J. and Sastry S. (1989): Dynamic control of redundant manipulators. - Journal of Robotic Systems, vol.6, No.2, pp.133-148.

[3] Canudas C., Siciliano B. and Bastin G. (Eds. ) (1996): Theory of Robot Control. - London: Springer.

[4] Siciliano B., Sciavicco L., Villani L. and Oriolo G. (Eds.) (2010): Robotics: Modelling, Planning and Control. Springer Verlag.

[5] Galicki M. (2004): Path following by the end-effector of a redundant manipulator operating in a dynamic environment. - IEEE Transactions on Robotics, vol.20, No.6, pp.1018-1025.

[6] Kelly R. and Moreno J. (2005): Manipulator motion control in operational space using joint velocity inner loop. Automatica, vol.41, No.8, pp.1423-1432.

[7] Nakanishi J., Cory R., Mistry M., Peters J. and Schaal S. (2008): Operational space control: A theoretical and empirical comparison. - The International Journal of Robotics Research, vol.27, No.6, pp.737-757.

[8] Moreno-Valenzuela J. and Gonzales-Hernandez L. (2011): Operational space trajectory tracking control of robot manipulators endowed with a primary controller of synthetic joint velocity. - ISA Transactions, vol.50, No.1, pp.131140.

[9] Tatlicioglu E., Braganza D., Burg T.C. and Dawson D.M. (2008): Adaptive control of redundant robot manipulators with sub-task objectives. - In Proc. ACC, pp.856-860. 
[10] Sadeghian H., Keshmiri M., Villani L. and Siciliano B. (2012): Priority oriented adaptive control of kinematically redundant manipulators. - In Proc. IEEE RA, pp.293-298.

[11] Sadeghian H., Villani L., Kesmiri M. and Siciliano B. (2013): Dynamic multi-priority control in redundant robotic systems. - Robotica, vol.31, No.07, pp.1155-1167.

[12] Feng, G. and Palaniswami M. (1993): Adaptive control of robot manipulators in task space. - IEEE Trans. Automat. Contr., vol.38, No.1, pp.100-104.

[13] Zergeroglu E., Dawson D.M., Walker I. and Behal A. (2000): Nonlinear tracking control of kinematically redundant robot manipulators. - In Proc. ACC, vol.4, pp.2513-2517.

[14] Braganza D., Dixon W.E., Dawson D.M. and Xian B. (2005): Tracking control for robot manipulators with kinematic and dynamic uncertainty. - In Proc. CDC, pp.5293-5297.

[15] Braganza D., Dixon W.E., Dawson D.M. and Xian B. (2008): Tracking control for robot manipulators with kinematic and dynamic uncertainty. - International Journal of Robotics and Automation, vol.23, No.2, pp.117-126.

[16] Galicki M. (2007): Adaptive path-constrained control of a robotic manipulator in a task space. - Robotica, vol.25, No.1, pp.103-112.

[17] Cheah C.C., Liu C. and Slotine J.J. (2006): Adaptive tracking control for robots with unknown kinematic and dynamic properties. - The International Journal of Robotics Research, vol.25, No.3, pp.283-296.

[18] Li X. and Cheah C.C. (2012): Adaptive regional feedback control of robotic manipulator with uncertain kinematics and depth information. - In Proc. ACC, pp.5472-5477.

[19] Li X. and Cheah C.C. (2013): Global task-space adaptive control of robot. - Automatica, vol.49, No.1, pp.58-69.

[20] Galicki M. (2013): Inverse-free control of a robotic manipulator in a task space. - Robotics and Autonomous Systems, vol.62, No.2, pp.131-141.

[21] Zergeroglu E., Sahin H.T., Ozbay U. and Tektas H.A. (2006): Robust tracking control of kinematically redundant robot manipulators subject to multiple self-motion criteria. - In Proc. IEEE Control Appl., pp.2860-2865.

[22] Ozbay U., Sahin H.T. and Zergeroglu E. (2008): Robust tracking control of kinematically redundant robot manipulators subject to multiple self-motion criteria. - Robotica, vol.26, No.06, pp.711-728.

[23] Singh H.P. and Sukavanam N. (2012): Neural network based control scheme for redundant robot manipulators subject to multiple self-motion criteria. - Mathematical and Computer Modelling, vol.55, No.3, pp.1275-1300.

[24] Shamir T. and Yomdin Y. (1988): Repeatability of redundant manipulators: Mathematical solution of the problem. IEEE Trans. Automat. Cont., vol.33, No.11, pp.1004-1009.

[25] Roberts R.G. and Maciejewski A.A. (1992): Nearest optimal repeatable control strategies for kinematically redundant manipulators. - IEEE Trans. Robot. Automat. vol.8, No.3, pp.327-337.

[26] Nakamura Y. and Hanafusa H. (1986): Inverse kinematic solutions with singularity robustness for robot manipulator control. - Dyn. Syst. Measurements and Control, vol.108, No.3, pp. 163-171.

[27] Wampler C. W. and Leifer L. J. (1988): Applications of damped least-squares methods to resolved-rate and resolved-acceleration control of manipulators. - J. Dyn. Syst. Measurements and Control, vol.110, No.1, pp.3138.

[28] Seraji H. and Colbaugh R. (1990): Improved configuration control for redundant robots. - J. Robot. Syst., vol.7, No.6, pp.897-928.

[29] Peng Z.X. and Adachi N. (1993): Compliant motion control of kinematically redundant manipulators. IEEE Trans. Robot. Automat., vol.9, No.6, pp.831-836.

[30] Ott C., Dietrich A. and Schaffer A.A. (2015): Prioritized multi-task compliance control of redundant manipulators. - Automatica, vol.53, pp.416-423.

[31] Oh Y. and Chung W.K. (1999): Disturbance observer based motion control of redundant manipulators using inertially decoupled dynamics. - IEEE/ASME Trans. Mechatronics, vol.4, No.2, pp.133-146. 
[32] Colbaugh R. and Glass K. (1995): Robust adaptive control of redundant manipulators. - J. Intell. Robot. Syst., vol.14, No.1, pp.69-88.

[33] Balleieul J. (1985): Kinematic programming alternatives for redundant manipulators. - In Proc. IEEE Int. Conf. on Robotics and Automation, vol.2, pp.722-728.

[34] Wolovich W.A. and Elliot H. (1984): A computational technique for inverse kinematics. - In Proc. 23rd IEEE Conference on Decision and Control, pp.1359-1363.

[35] Siciliano B. (1990): A closed-loop inverse kinematic scheme for on-line joint-based robot control. - Robotica, vol.8, No.3, pp.231-243.

[36] Kelly R. (1996): Robust asymptotically stable visual servoing of planar robots. - IEEE trans. Rob. Automat., vol.12, No.5, pp.759-766.

[37] Cheah C.C. (2006): On duality of inverse Jacobian and transpose Jacobian in task-space regulation of robots. In Proc. IEEE Int. Conf. on Robotics and Automation, pp.2571-2576.

[38] Cheah C.C., Lee K., Kawamura S. and Arimoto S. (2000): Asymptotic stability control with approximate Jacobian matrix and its application to visual servoing. - In Proc. IEEE Decision and Control, pp.3939-3944.

[39] Moosavian S.A.A. and Papadopoulos E. (2007): Modified transpose Jacobian control of robotic systems. Automatica, vol.43, No.7, pp.1226-1233.

[40] Edwards C. and Spurgeon S.K. (1998): Sliding Mode Control: Theory and Application. - Taylor and Francis: London.

[41] Utkin V.I. (1992): Sliding modes in Optimization and Control Problems. - New York: Springer.

[42] Fridman L. (2002): Singularly perturbed analysis of chattering in relay control systems. - IEEE Transactions on Automatic Control, vol.47, No.12, pp.2079-2084.

[43] Galicki M. (2015): Finite-time control of robotic manipulators. - Automatica, vol.51, pp.49-54.

[44] Galicki M. (2016): Finite-time trajectory tracking control in a task space of robotic manipulators. - Automatica, vol.67, pp.165-170.

[45] Galicki M. (2016): Robust task space finite-time chattering-free control of robotic manipulators. - J. Intell. Robot Syst., DOI: 10.1007/s10846-016-0387-3.

[46] Haessing D. and Friedland B. (1991): On the modeling and simulation of friction. - Transactions of the ASME, Journal of Dynamic Systems, Measurements and Control, vol.113, No.3, pp.354-362.

[47] Wit C., Ollson H., Astrom K. and Lischinsky P. (1995): A new model for control of systems with friction. - IEEE Trans. Automat. Contr., vol.40, No.3, pp.419-425.

[48] Han J.D., He Y.Q., Xu W.L. (2007): Angular acceleration estimation and feedback control: An experimental investigation. - Mechatronics, vol.17, No.9, pp.524-532.

[49] Spong M.W. and Vidyasagar M. (1989): Robot Dynamics and Control. - New York: Wiley.

[50] Yoshikawa T. (1985): Manipulability of robotic mechanisms. - Int. J. Robotics Res., vol.4, No.2, pp.3-9.

[51] Maciejewski A.A. and Klein C.A. (1985): Obstacle avoidance for kinematically redundant manipulators in dynamically varying environments. - I. J. Rob. Res., vol.4, No.3, pp.109-117.

[52] Perdereau V., Passi C. and Drouin M. (2002): Real-time control of redundant robotic manipulators for mobile obstacle avoidance. - Robotics and Autonomous Systems, vol.41, No.1, pp.41-59.

[53] Cruse H. et al. (1990): On the cost functions for the control of the human arm movement. - Biological Cybernetics, vol.62, No.6, pp.519-528.

[54] Feng Y., Yu X. and Man Z. (2002): Non-singular terminal sliding mode control of rigid manipulators. Automatica, vol.38, No.12, pp.2159-2167. 
[55] Yu S., Yu X., Shirinzadeh B. and Man Z. (2005): Continuous finite-time control for robotic manipulators with terminal sliding mode. - Automatica, vol.41, No.11, pp.1957-1964.

[56] Zhao D., Li S. and Gao F. (2009): A new terminal sliding mode control for robotic manipulators. - International Journal of Control, vol.82, No.10, pp.1804-1813.

[57] Bhat S.P. and Bernstein D.S. (2000): Finite-time stability of continuous autonomous systems. - SIAM Journal on Control and Optimization, vol.38, No.3, pp.751-766.

[58] Hong Y.G. (2002): Finite-time stabilization and stabilizability of a class of controllable systems. - Systems Control Letters, vol.46, No.4, pp.231-236.

[59] Bartolini G., Ferrara A. and Punta E. (2000): Multi-input second-order sliding-mode hybrid control of constrained manipulators. - Dynamics and Control, vol.10, No.3, pp.277-296.

[60] Bartolini G., Ferrara A., Usai E. and Utkin V.I. (2000): On multi-input chattering-free second-order sliding mode control. - IEEE Transactions on Automatic Control, vol.45, No.9, pp.1711-1717.

[61] Bartolini G., Pisano A., Punta E. and Usai E. (2003): A survey of applications of second-order sliding mode control to mechanical systems. - International Journal of Control, vol.76, No.9-10, pp.875-892.

[62] Ferrara A. and Capisani L.M. (2011): Second order sliding modes to control and supervise industrial robot manipulators. - In. L. Fridman et al. (Eds.): Sliding modes, LNCIS, Lecture Notes in Control and Information Sciences, vol.412, pp.541-567.

[63] Filippov A.F. (1988): Differential Equations with Discontinuous Right-hand Side. - Dordrecht, Netherlands.

[64] Canudas de Wit C., Fixot N. and Astrom K.J. (1992): Trajectory tracking in robot manipulators via nonlinear estimated state feedback. - IEEE Trans. Robot. Automat., vol.8, No.1, pp.138-144.

[65] ElBeheiry E.M., Zaki A. and ElMaraghy W.H. (2003): A unified approach for independent manipulator joint acceleration control and observation. - ASME Dynamic Systems and Control Division, vol.72, No.1, pp.659-666.

[66] Khalil H.K. and Praly L. (2014): High-gain observers in nonlinear feedback control. - Int. J. Robust and Nonlinear Control, vol.24, No.6, pp.993-1015.

[67] Ball A.A. and Khalil H.K. (2013): A nonlinear high-gain observer for systems with measurement noise. - IEEE Trans. Automat. Control, vol.58, pp.569-580.

[68] De Luca A., Schroder D. and Thummel M. (2007): An acceleration-based state observer for robot manipulators with elastic joints. - In Proc. IEEE International Conference on Robotics and Automation, pp.3817-3823.

[69] Hsiao T. and Weng M.C. (2013): Robust joint position feedback control of robot manipulators. - J. Dynam. Syst., Measurement, and Control, vol.135, No.3, 031010.

[70] Davila J., Fridman L. and Levant A. (2005): Second-order sliding mode observer for mechanical systems. - IEEE Trans. Automat. Control, vol.50, No.11, pp.1785-1789.

[71] Atasi A.N. and Khalil H.K. (2000): Separation results for the stabilization of nonlinear systems using different high-gain observer designs. - Systems and Control Letters, vol.39, No.3, pp.183-191.

[72] Levant A. and Livne M. (2012): Exact differentiation of signals with unbounded higher derivatives. - IEEE Transactions on Automatic Control, vol.57, No.4, pp.1076-1080.

[73] Levant A. (2003): Higher-order sliding modes, differentiation and output-feedback control. - International Journal of Control, vol.76, No.9-10, pp.924-941.

Received: June 13, 2017

Revised: September 19, 2017 